\title{
On the power allocation strategies in coordinated multi-cell networks using Stackelberg game
}

\author{
Shima Haddadi ${ }^{1}$, Ashkan Oliaiee ${ }^{1}$, Hamid Behroozi ${ }^{1 *}$ and Babak Hossein Khalaj ${ }^{1,2}$
}

\begin{abstract}
In this paper, we study the power allocation problem in multi-cell OFDMA networks, where given the tradeoff between user satisfaction and profit of the service provider, maximizing the revenue of the service provider is also taken into account. Consequently, two Stackelberg games are proposed for allocating proper powers to central and cell-edge users. In our algorithm, assuming the fact that users agree to pay more for better QoS level, the service provider imposes optimum prices for unit-power transmitted to users as they request different levels of QoS. In addition, in order to improve system performance at cell-edge locations, users are divided into two groups based on their distance to the corresponding base-station (BSs): Central users and cell-edge users. The paper also exploits unique features of Coordinated Multi-Point Joint Transmission (CoMP-JT) where coordinated BSs are clustered together statically or dynamically in order to also address the requirements of cell-edge users. After simulating the proposed game with static clustering, a simple dynamic clustering algorithm is introduced for inter-cell coordinated networks where its performance is evaluated through simulations.
\end{abstract}

Keywords: Game theory, Inter-cell interference coordination (ICIC), Coordinated multi-point joint transmission, Resource allocation problem, Static and dynamic clustering, Power consumption

\section{Introduction}

\subsection{Background and related works}

Satisfying the growing demand for mobile services and radio communications makes future wireless networks to fully reuse resources such as available frequency bands. This increase in use of resources in turn leads to high increase in inter-cell interference (ICI) level. Increased interference levels degrade the system performance in serving users and due to the near-far effects on the signal-to-interference-plus-noise ratio (SINR) level, users in cell-edges are more sensitive to high interference than central ones. ICI mitigation along with demand for higher data-rate requires utilization of advanced technologies. Subsequently, resource management plays a major role in next generation mobile networks.

${ }^{*}$ Correspondence: behroozi@sharif.edu

${ }^{1}$ Department of Electrical Engineering, Sharif University of Technology, Tehran, Iran

Full list of author information is available at the end of the article
In Long Term Evolution (LTE) standard, developed by the 3rd Generation Partnership Project (3GPP) standardization body, three general approaches are proposed to overcome inter-cell interference effect: ICI randomization, ICI cancellation and ICI coordination (ICIC) [1-3]. Our research is based on the last approach which has lower computational complexity. The ICIC approach tries to improve the cell-edge performance by accepting some level of interference and thus provides more homogeneous service to users located at different regions of the network. Fractional Frequency Reuse (FFR) is an asserted idea in this context that divides available frequency bands into different groups, where each band is designed to be dedicated to certificated users with different reuse factors which correspond to their channel conditions [4]. Consequently, quality of communication of users who suffer more from interference can be improved by less reuse of their allocated subchannels.

Another promising technology to improve the conditions at cell-edges is coordinated multi-point joint transmission (CoMP-JT) [5]. In cooperative networks, in order

\section{Springer}


to eliminate the interference on cell-edge users, a set of coordinated BSs which are clustered together serve one cell-edge user simultaneously over the same resource [6]. It is well-known that better performance can be achieved by increasing the number of cells that are grouped together (called the cluster size). However, in reality, only a limited number of BSs can coordinate the transmission [7]. This raises the question that coordinating transmission among which selection of BSs will lead to a more efficient CoMP-JT implementation. In practice, cells can form clusters either statically or dynamically [8]. Static clusters are constructed according to the geographical criteria. Consequently, they are fixed for all users and are not changed over time. On the other hand, adaptive clustering applies real-time cluster reformation by perceiving the condition of each user at each time-instant. In fact, variations in system loading or mobility of users may lead to new clustering architecture. Naturally, dynamic clustering can improve system performance noticeably at the price of higher system complexity.

Although CoMP-JT can enhance the throughput of users located in cell-edges noticeably, it also increases the complexity, signaling overhead on backhaul network which is commonly known as X2 interface and information exchange among cells. The authors in [5] brought some practical techniques to overcome the fundamental requirements of CoMP-JT. In addition, field trial results of [2, 9-11] have confirmed the advantages of applying CoMP in practical systems. So in this paper, these preliminaries are assumed to be provided. We also ignore the imposed complexity caused by backhaul requirements of CoMP and assume the channel gains between users and BSs to be properly estimated.

Joint multi-cell coordinated scheduling and multipleinput multiple-output (MIMO) techniques are also well-known schemes applied in LTE [12]. A multicell cooperative zero-forcing beamforming (ZFBF) scheme is proposed in [13] in which all BSs use CoMP transmission to maximize the energy efficiency (i.e., the average datarate per unit power). A location-aware multicell cooperation scheme for downlink transmission in orthogonal frequency-division multiple access (OFDMA)-based networks is proposed in [14] which only uses CoMP transmission to serve users with low SINR level while users with acceptable SINR conditions are served by a single BS.

The efficiency of a wireless cellular network mostly depends on allocating schemes for valuable resources such as power and bandwidth. Providing a minimum quality of service (QoS) level for all users, minimizing power consumption and maximizing the difference between the benefits and costs of the network for one user result in a typical choice of metrics used to allocate resources among different users. It should be noted that in this paper, we choose the requested SINR of each user as the QoS index.

Resource allocation problem exists in both single-cell and multi-cell scenarios [15]. As shown in Fig. 1a, interactions of cells in the single-cell scenario are limited to the interference signal caused by other users when no clustering is applied. However, for the multi-cell scenario which is illustrated in Fig. 1b, cells are clustered to apply COMPJT technique. While the first scenario enables the network to serve more users, applying the second approach yields to a better communication quality for cell-edge users, which naturally forces additional complexity to the overall system.

Despite optimality of centralized techniques in resource allocation, finding the analytic formulation and in turn the optimum point is prohibitively difficult due to the non-convexity of the associated optimization problem $[16,17]$. Furthemore, the resource allocation problem in CoMP is proved to be NP-complete [18]. Game theory which covers such scenarios is a powerful tool in providing sub-optimal solutions to model and analyze resource allocation problems in a distributed way.

In recent years, various non-cooperative games are proposed in the literature, trying to maximize the utility of selfish users [19-23]. Also, [24] presents a resource allocation based on cooperative game. But, in addition to the user satisfaction, profit of the service provider is another important factor that should be considered in the network. Hierarchical games such as Stackelberg

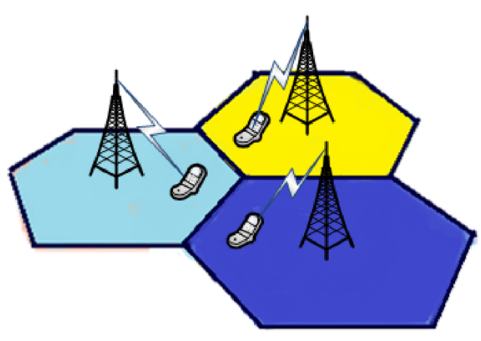

(a) Single-cell scenario

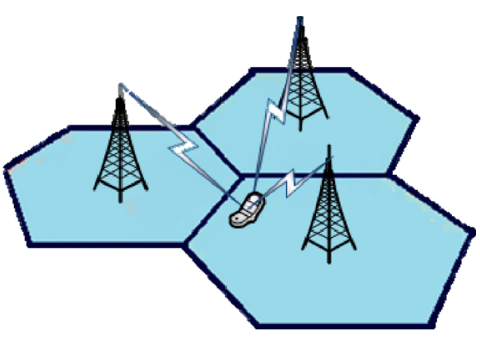

(b) Multi-cell scenario.

Fig. 1 Different scenarios for the resource allocation problem. a Single-cell scenario, where interactions of cells are limited to the interference signal caused by other users when no clustering is applied. b Multi-cell scenario, where cells are clustered to apply COMP JT technique 
or leader-follower game can properly cover the pricingbased interactions between users and the service provider [25]. Namely, the service provider determines differential prices per unit of transmitting power-level for each user, proportional to the requested service of user, in order to maximize its revenue from the network. On the other hand, users (which are also called followers) adjust their power levels to get maximum services from the network with the least imposed costs. In [26], the authors try to model the pricing-based interactions between secondary and primary users in a time-division multiple access (TDMA) cooperative cognitive-radio networks using Stackelberg game. Furthermore, [27] presents a Stackelberg game to model the power allocation of codedivision multiple access (CDMA) cognitive-radio network in uplink side. However, key issues such as power consumption and QoS of users are addressed in none of these references. Another Stackelberg game proposed for the same network in the downlink side [28] also does not taken QoS into account.

Various algorithms for static clustering were already presented in earlier works [29-31]. However, due to their limited performance gain, dynamic clustering has attracted more attention in recent years [32-35]. For example, the work in [32] is based on the graph theory with the aim of maximizing the sum-capacity. Indeed, each BS will be associated with the cluster which has the maximum joint capacity rate with other BSs belonging to that cluster. In [33] cells are clustered dynamically according to whether perfect channel state information (CSI) is available or not. In [34], a co-channel inter-cluster interference canceler is proposed for MIMO networks by performing linear processing combined with multiuser beamforming. Also, the authors in [35] introduce overlapping clustering. Requiring high level of computational complexity is the major drawback of the aforementioned algorithms. In addition, reference signal receiving power (RSRP) is used in $[36,37]$ to select cooperating BSs. The authors in [37] propose a resources allocation algorithm based on non-cooperative game considering the throughput of users and their QoS to evaluate the system performance.

\subsection{Motivations, results, and paper organization}

To the best of our knowledge, the main challenge of future networks is the ability to satisfy the growing and high quality demands of users for various kind of services even in cell-edges while their revenue is also maximized. Consequently, the first novelty of our approach compared with aforementioned studies is that we allow users to request different QoS levels due to their interest for different services and of course their affordable payment. Since CoMP-JT is a key technology to cope with high quality demands of cell-edge users and no revenue maximization game model that incorporates such characteristics in coordinated multi-cell networks is yet applied, the main motivation in this paper is proposition of a Stackelberg game for OFDMA multi-cell cellular networks using a combination of CoMP-JT technologies and FFR technique. We also compare static and dynamic clustering and analyze their effects in terms of system performance. Subsequently, a simple dynamic clustering algorithm is proposed in order to verify the effects of clustering model in system performance.

Our earlier results show that the proposed power allocation algorithm can properly satisfy even high quality demands of users near to BSs. In contrast, the expanded version of our game to apply CoMP-JT demonstrates that static BS selection is only capable of affording high QoS level for cell-edge users in special locations. Finally, by proposing a simple dynamic clustering method, the performance of system in cell-edges can be noticeably improved.

The remainder of this paper is organized as follows: The proposed architecture for the system model is described in Section 2. Section 3 focuses on the power allocation strategies of users near to the center of cells (central users) and the extension of the proposed game to apply COMP JT for cell-edge users is provided in Section 4. Both proposed power allocation algorithms are simulated in hypothetical static clusters in Section 5. Presented results show how CoMP-JT helps to improve cell-edge performance. In Section 6, a dynamic clustering algorithm with the aim of performance improvement by imposing low-level of complexity to the system is introduced. Simulation results for the proposed method of clustering are presented in Section 6.3 in which performance improvement in comparison with the static clustering is evaluated. Finally, Section 7 concludes the paper.

\section{Proposed system architecture}

An OFDMA cellular network, similar to what is shown in Fig. 2, consists of $M$ cells is assumed. All cells are partitioned into $C$ clusters each containing $B$ cells. One basestation is assumed to be located in the middle of each cell, providing communication services to users in the downlink side. In the proposed architecture, each cell is sectorized into two concentric regions: central and cell-edge regions. Consequently, using FFR technique, some frequencies are reserved for central users of each cell. Central users are expected to receive services only from the BS of their own cells with frequency reuse factor of 1 . On the other hand, each user in the cell-edge is served not only by the BS of its cell, but also by all BSs belonging to its hypothetical static cluster or dynamic-formed one, in order to mitigate its higher level of ICI. As a result, frequency reuse factor of bands in cell-edge regions will be equal to 


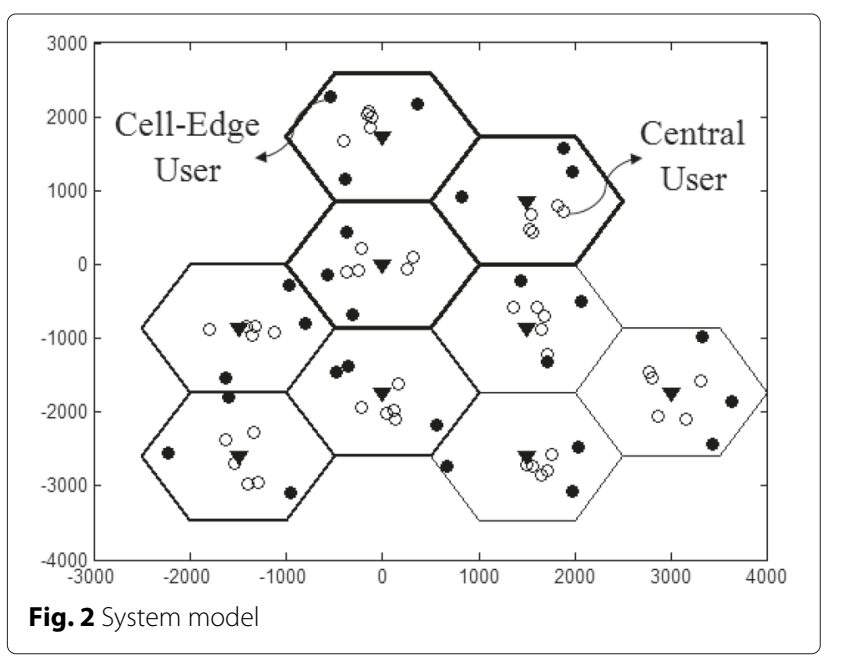

1 in each cluster. It is assumed that users are located randomly in central and cell-edge regions and each of them can use only one of the available channels. Allocated channels to central users can be reused in other cells, while cell-edge channels are only allowed to be reused in other clusters. Consequently, we have inter-cell interference for central users and inter-cluster interference for cell-edge users. Maximum affordable transmitting power on each channel by each BS is limited to $p_{\max }$. Noise variance on each channel is also assumed to be $\sigma_{0}^{2}$. Additionally, each user and each BS can obtain the needed channel information via existing control stations in the network. We also consider stationary mobile stations where assuming fixed channel gains over the time is reasonable.

Meanwhile, in this paper, we intend to provide dynamic SINR thresholds for all users. In order to reach this goal, we consider a minimum SINR threshold $\gamma_{t h}$ for all users in the network. The requested SINR threshold for user $i$ in central region will be $\alpha_{i, m} \gamma_{t h}$ and in cell-edge region will be $\alpha_{i, c} \gamma_{t h}$, where the coefficient $\alpha_{i, m}$ will be determined by central user $i$ in cell $m$ and the coefficient $\alpha_{i, c}$ will be assigned by cell-edge user $i$ in cluster $c$.

By sectorizing each cell, indeed, for central users we are facing to single-cell scenario of resource allocation while multi-cell scenario should be applied for cell-edge users. In our investigation, first we focus on resource allocation problem in central region with single-cell approach, then try to exploit COMP JT improvements using multi-cell scenario.

\section{Power allocation strategy for central users}

In this section, we focus on serving users located in central region. After defining the required parameters, the proposed Stackelberg game formulation in non-cooperative framework is determined. Eventually, convergence and uniqueness of the Nash equilibrium point for this proposed game is evaluated.

\subsection{Problem definitions}

Required parameters for system model in central region are described in Table 1.

In this case, user $i$ in cell $m$ on channel $l_{i}$ is exposed to the total interference signal equal to:

$$
I_{i, l_{i}}^{m}=\sum_{j=1, j \neq m}^{M} p_{i, l_{i}}^{m, j} h_{i, l_{i}}^{m, j} .
$$

So, the SINR level of such user will be determined as:

$$
\gamma_{i, l_{i}}^{m}=\frac{p_{i, l_{i}}^{m, m} h_{i, l_{i}}^{m, m}}{I_{i, l_{i}}^{m}+\sigma_{0}^{2}}=\frac{p_{i, l_{i}}^{m, m} h_{i, l_{i}}^{m, m}}{I_{I, N}^{i, m}},
$$

where $I_{I . N \text {. }}^{i, m}$ refers to the summation of noise power and the received interference power at user $i$ in cell $m$ on channel $l_{i}$, caused by users of other cells using the same channel.

\subsection{Stackelberg game model for central users}

We are interested in developing a Stackelberg game which is implemented in a distributed way. For this purpose, we determine a normal game as $G=(\mathcal{N}, \mathcal{A}, \mathcal{U})$ where:

- $\mathcal{N}=\{1, \ldots, n\}$ denotes the set of players or decision makers. Due to our Stackelberg game model in central region, this set consists of central users and BSs.

- $\mathcal{A}=\left\{A_{1}, \ldots, A_{n}\right\}$ determines the set of possible actions that each player can choose as its strategy. This set is referred to as the strategy space. Each Stackelberg game consists of users strategies as well as BSs strategies.

- The main part of each game is the set of utility functions which is defined according to player's preferences. In each step of the game, each player chooses the strategy from its strategy space which maximizes its objectives. In our model, each BS wants

Table 1 System parameters in central region

\begin{tabular}{ll}
\hline Parameter & Definition \\
\hline$N_{i n t}$ & $\begin{array}{l}\text { Number of users located randomly in central region } \\
\text { of each cell }\end{array}$ \\
$M$ & Number of cells \\
$\sigma_{0}^{2}$ & Additive noise power \\
$h_{i, l_{i}}^{m, j}$ & Channel gain between user $i$ of cell $m$ on channel $l_{i}$ \\
$p_{i, l_{i}}^{m, j}$ & and BS of cell $j$ \\
$p_{i, l_{i}}^{m, m}$ & Transmitted power from BS of cell $j$ to user $i$ of cell $m$ \\
$\gamma_{i, l_{i}}^{m}$ & on channel $l_{i}$ \\
$\gamma_{t h}$ & Transmitted power to user $i$ of cell $m$ on channel $l_{i}$ \\
$p_{\text {max }}$ & from BS of its cell \\
\hline
\end{tabular}


to earn higher income from users by consuming as low-level of power as possible, while each user is interested in gaining higher level of SINR or equivalently higher data-rate while paying less to the service provider.

Stackelberg games can be solved by using backward induction. In fact, first each user considering fixed unit-prices for power transmitted from BSs, maximizes its utility function and choose its optimum power from the user's strategy space. Then, each BS, considering required transmitting powers, maximizes its revenue to determine the best price for each user's unit-power.

Consequently, the utility function of user $i$ from cell $m$ which uses channel $l_{i}$ can be defined as:

$$
\begin{aligned}
U_{i}^{m}= & \alpha_{i, m}^{2} \operatorname{sgn}\left(\gamma_{i, l_{i}}^{m}-\alpha_{i, m} \gamma_{t h}\right) \log \left(1+\gamma_{i, l_{i}}^{m}\right) \\
& -\left(\lambda_{i}^{m} p_{i, l_{i}}^{m, m}+\varphi_{i}^{m}\right)+\frac{\gamma_{i, l_{i}}^{m}}{\alpha_{i, m} \gamma_{t h}} \mathrm{u}\left(\gamma_{i, l_{i}}^{m}-\alpha_{i, m} \gamma_{t h}\right),
\end{aligned}
$$

where $\lambda_{i}^{m}$ and $\varphi_{i}^{m}$ are respectively the unit-price and the subscription cost that $\mathrm{BS}$ of cell $m$ imposes to user $i$ located in this cell. The first term of (3) is proportional to the received rate at user $i$ of cell $m$ and the second term is the imposed cost of serving BS to such user. The third term and also the coefficient of the rate in the first term are adopted to ensure the SINR of user $i$ to be more than its demand. Indeed, the objectives of user $i$ are considered as follows: 1) Ensuring that its received SINR is larger than or equal to its target SINR, 2) maximizing its data sum-rate and 3) paying the lowest price to the service provider. Also, in (3), $\alpha_{i, m}$ is the factor that shows the willing of $i^{\text {th }}$ user to pay more in lieu of getting better quality of services.

On the other hand, from the perspective of revenue maximization, the object function for each BS can be formulated as:

$$
U_{B S}^{m}=\sum_{i=1}^{N_{\text {int }}}\left(\lambda_{i}^{m} p_{i, l_{i}}^{m, m}+\varphi_{i}^{m}\right)-\sum_{i=1}^{N_{\text {int }}}\left(\gamma_{i, l_{i}}^{m}-\alpha_{i, m} \gamma_{t h}\right)^{2}
$$

In this utility function, the first term denotes the receiving costs of BS $m$ from all users in the same cell. In order to keep the required power-level of each user as low as possible, we add the second term to each BS's objectives. In this case, there will be no incentive for the service provider to provide a QoS better than what is required. Therefore, the power consumption could reach to the minimum possible level.

Additionally, not only the location of users should not have any effect on $\varphi_{i}^{m}$, but also we expect $\varphi_{i}^{m}$ and $\lambda_{i}^{m}$ to increase when higher $\mathrm{QoS}$ is requested. By taking attention to the relation of unit-price and channel condition in [27], we define the subscription $\operatorname{cost} \varphi_{i}^{m}$ as a function of $\lambda_{i}^{m}$ as the following:

$$
\varphi_{i}^{m}=\lambda_{i}^{m} \frac{I_{I . N .}^{i, m}}{h_{i, l_{i}}^{m, m}} .
$$

\subsubsection{Nash equilibrium of proposed game}

a) Main concepts of Nash equilibrium

Generally, the main goal of any hierarchical game is achieving the Nash equilibrium point which is defined as the profile of optimal actions for users. On the other hand, it is the point that there is no incentive for each player to deviate from its choice to have extra profile [38]. Mathematically, in our game, a strategy set is defined as a Nash equilibrium point if we have:

$$
\begin{gathered}
U_{i}^{m}\left(p_{i, l_{i}}^{*}\right) \geq U_{i}^{m}\left(p_{i, l_{i}}\right) \quad \forall\left(p_{i, l_{i}}\right), i=1, \ldots, N \\
U_{B S}^{m}\left(\lambda^{*}, \boldsymbol{P}^{*}\right) \geq U_{B S}^{m}(\lambda, \boldsymbol{P}) \quad \forall(\boldsymbol{\lambda}, \boldsymbol{P}), m=1, \ldots, M
\end{gathered}
$$

where $\boldsymbol{P}^{*}=\left[p_{1, l_{1}}^{*}, p_{2, l_{2}}^{*}, \ldots, p_{N, l_{N}}^{*}\right]$ and similarly $\lambda^{*}=$ $\left[\lambda_{1}^{*}, \lambda_{2}^{*}, \ldots, \lambda_{N}^{*}\right]$

In order to solve a Stackelberg game, Nash equilibrium of the subgame should be obtained by calculating the best responses of players using backward induction. Namely, the leader first predicts the response of the follower which is considered to be rational and then chooses a strategy which maximizes its payoff. Sequentially, the follower chooses the anticipated response to the observed strategy of the leader.

Note 1: In principles of game theory, according to "Kakutani Fixed Point Theorem" , a game should meet two following conditions to have a pure Nash equilibrium point [38]:

1. The strategy space should be nonempty, closed, bounded and also a convex set.

2. The utility function should be not only continuous in strategy space but also be a concave function.

Remark 1. A set is said to be convex if and only if with the assumption of $0 \leq \theta \leq 1$ and having $x_{1}$ and $x_{2}$ as points in predetermined set, $y=\theta x_{1}+(1-$ $\theta) x_{2}$ belongs to the set too [39]. Also a function $f(x)$ is concave if it satisfies :

$$
\frac{\partial^{2} f(x)}{\partial x^{2}} \leq 0
$$

b) Existence of a Nash equilibrium point in the proposed Stackelberg game

According to system's specified parameters, the strategy space of the proposed game would be the following set: $\left[0, p_{\max }\right]$. It is obvious that this set is 
non-empty, closed and bounded. Hence, for satisfying the first condition of Note 1 , the convexity of the strategy set should be proved. Assuming $p_{1}$ and $p_{2}$ as two allocated powers from the strategy space $\left[0, p_{\max }\right]$, we have:

$$
0 \leq \theta p_{1}+(1-\theta) p_{2} \leq p_{\max } .
$$

So, the strategy space is convex.

Based on Note 1, in addition to the abovementioned condition, concavity of utility functions with respect to their own strategies should exist. By calculating the second-order partial derivative of (3) with respect to $p_{i, l_{i}}^{m, m}$, we obtain:

$$
\frac{\partial^{2} U_{i}^{m}}{\partial\left(p_{i, l_{i}}^{m, m}\right)^{2}}=-\frac{\alpha_{i, m}^{2} h_{i, l_{i}}^{m, m}}{I_{I . N .}^{i, m}\left(1+\gamma_{i, l_{i}}^{m}\right)^{2}}
$$

Since (9) is negative, the utility function of each central user is always strictly concave.

For utility function of BSs, expressed in (4), to be concave, we need to have:

$$
\lambda_{i}^{m}<\frac{6 \alpha_{i, m}^{3} \gamma_{t h} h_{i, l_{i}}^{m, m}}{I_{I . N .}^{i, m}\left(1+4 \alpha_{i, m} \gamma_{t h}+4 \alpha_{i, m}^{2} \gamma_{t h}^{2}\right)}+\frac{h_{i, l_{i}}^{m, m}}{I_{I . N .}^{i, m} \alpha_{i, m} \gamma_{t h}},
$$

Satisfying (10) indicates that each proposed game has at least one pure Nash equilibrium point.

c) Calculating Nash equilibrium point of the proposed game

According to the discussed principle of Stackelberg game, in order to obtain the unique Nash equilibrium point for the proposed games, the transmitting power for each user should be optimized using (11), considering unit prices as fixed parameters:

$$
\frac{\partial U_{i}^{m}}{\partial p_{i, l_{i}}^{m, m}}=0 \text {. }
$$

In maximizing procedure of (3), two cases should be considered, $\gamma_{i, l_{i}}^{m}<\alpha_{i, m} \gamma_{t h}$ and $\gamma_{i, l_{i}}^{m} \geq \alpha_{i, m} \gamma_{t h}$. In the first case, the objective function of user $i$ defined in (3) will be always non-positive, so the maximum utility that is equal to zero will be occurred when user's power becomes zero as well. Now, considering $\gamma_{i, l_{i}}^{m} \geq \alpha_{i, m} \gamma_{t h}$, the optimal power allocation strategy for central user $i$ will be obtained as the following:

$$
p_{i, l_{i}}^{m, m}\left(\lambda_{i}^{m}\right)^{(k+1)}=\frac{\alpha_{i, m}^{2}}{\lambda_{i}^{m(k+1)}-\frac{h_{i, l_{i}}^{m, m}}{I_{I . N .}^{i, m(k)} \alpha_{i, m} \gamma_{t h}}}-\left.\frac{I_{I . N .}^{i, m}(k)}{h_{i, l_{i}}^{m, m}}\right|_{0} ^{P_{\max }}
$$

In the next step, we substitute (12) in BS objective functions for central users in (4). Then, by maximizing the utility function of the BS, optimum unit-price of user $i$ in central region of cell $m$ is calculated as:

$$
\lambda_{i}^{m(k+1)}=\frac{4 \alpha_{i, m}^{3} \gamma_{t h} h_{i, l_{i}}^{m, m}}{I_{I . N .}^{i, m(k)}\left(1+4 \alpha_{i, m} \gamma_{t h}+4 \alpha_{i, m}^{2} \gamma_{t h}^{2}\right)}+\frac{h_{i, l_{i}}^{m, m}}{\alpha_{i, m} \gamma_{t h} I_{I . N .}^{i, m}(k)},
$$

Indeed, as we expected, not only the values of $\varphi_{i}^{m}$ is constant during all iterations and have no dependance on user's locations, but also the service provider considers higher unit-prices and subscription costs for central users requesting higher levels of QoS.

Furthermore, it is obvious that (13) satisfies the required condition of having unique Nash equilibrium point, provided in (10). Moreover, by substituting (13) in (12), it can be easily proved that obtained SINR of user $i$ is always more than its target with a small deviation

d) Uniqueness of obtained Nash equilibrium point

In [40], for an utility based iterative algorithm determined as $p_{i}^{(k+1)}=f_{i}\left(p_{i}^{(k)}\right)$ to converge to a unique Nash equilibrium point, the author has pointed that the updating function $f_{i}$ should be:

$$
\begin{aligned}
\text { - } & \text { Positive. i.e. } f_{i}\left(p_{i}^{(k)}\right) \geq 0 . \\
- & \text { Monotone which means } \\
& p_{i_{1}}^{(k)} \geq p_{i_{2}}^{(k)} \Rightarrow f_{i}\left(p_{i_{1}}^{(k)}\right) \geq f_{i}\left(p_{i_{2}}^{(k)}\right) . \\
- & \text { Scalable in the sense that } \\
& \rho f_{i}\left(p_{i}^{(k)}\right) \geq f_{i}\left(\rho p_{i}^{(k)}\right) \forall \rho \geq 1 .
\end{aligned}
$$

Considering proposed game and iterative power allocation calculated in (12), first condition for convergence of our algorithm will be satisfied if:

$$
\lambda_{i}^{m(k+1)} \leq\left(\alpha_{i, m}^{2}+\frac{1}{\alpha_{i, m} \gamma_{t h}}\right) \frac{h_{i, l_{i}}^{m, m}}{I_{I . N .}^{i, m}(k)}
$$

According to (13), Satisfying (14) coincides to $1+$ $4 \alpha_{i, m}^{2} \gamma_{t h}^{2} \geq 0$ which is evident.

Also, calculated updating function has monotonicity decreasing prosperity if its first deviation respect to imposed interference becomes non-negative that requires exactly the mentioned obvious equation.

Eventually, by multiplying the power level of all users by factor $\rho \geq 1$ we have:

$$
\forall 1 \leq i \leq N_{i n t}, 1 \leq m \leq M \quad I_{I . N .}^{i, m}(\boldsymbol{P}) \leq I_{I . N .}^{i, m}(\rho \boldsymbol{P})
$$

where $\boldsymbol{P}=\left\{p_{i, l_{i}}^{m, j} \mid \forall 1 \leq i \leq N_{\text {int }}, 1 \leq m, j \leq M\right\}$. In this case if we define:

$$
\mu_{i, m}=\frac{\alpha_{i, m}^{2}\left(1+4 \alpha_{i, m} \gamma_{t h}+4 \alpha_{i, m}^{2} \gamma_{t h}^{2}\right)}{4 \alpha_{i, m}^{3} \gamma_{t h} h_{i, l_{i}}^{m, m}}-\frac{1}{h_{i, l_{i}}^{m, m}}
$$


Positivity of transmitting power leads to $\mu_{i, m} \geq 0$, so we have:

$$
\mu_{i, m} I_{I . N .}^{i, m}(\rho \boldsymbol{P}) \leq \mu_{i, m} I_{I . N .}^{i, m}(\boldsymbol{P}) \leq \rho \mu_{i, m} I_{I . N .}^{i, m}(\boldsymbol{P})
$$

By satisfying the third condition as well, the uniqueness of obtained Nash equilibrium point is ensured.

\section{Power allocation problem for cell-edge users}

As it is mentioned, COMP JT is designed to be applied in cell-edge region to improve communication quality in cell-edges in which two or more coordinated BSs are employed to provide service for one cell-edge user, simultaneously. Indeed, despite central region, we are facing to multi-cell approach of power allocation problem for celledge users. Therefore, in this section, it is assumed that coordinated cells are clustered together statically. So after describing the system parameters of cell-edge region and the manner of using CoMP-JT technique, the proposed Stackelberg game of central region is extended applying COMP JT technique and finally after evaluating the existence and convergence of extended game, the allocated power to cell-edge users is calculated.

\subsection{Problem specific definitions}

The parameters of system model in cell-edge region which are required to extend our Stackelberg game are listed in Table 2.

The basic idea behind our study for cell-edge users to apply COMP JT is spreading their requested SINR among

Table 2 System parameters in cell-edge region

\begin{tabular}{|c|c|}
\hline Parameter & Definition \\
\hline Nout & $\begin{array}{l}\text { Number of users located randomly in cell-edge region } \\
\text { of each cell }\end{array}$ \\
\hline C & Number of clusters \\
\hline$B$ & Number of cells belonging to each cluster \\
\hline$\sigma_{0}^{2}$ & Additive noise power \\
\hline$h_{i, l_{i}}^{c, c, b}$ & $\begin{array}{l}\text { Channel gain between user } i \text { of cluster } c \text { on channel } I_{i} \\
\text { and } b^{\text {th }} \text { BS of its cluster }\end{array}$ \\
\hline$h_{i, l_{j}}^{c j, b}$ & $\begin{array}{l}\text { Channel gain between user } i \text { of cluster } c \text { on channel } I_{i} \\
\text { and } b^{\text {th }} \text { BS of cluster } j\end{array}$ \\
\hline$p_{i, l_{i}}^{c, c, b}$ & $\begin{array}{l}\text { Transmitted power from BS } b \text { of cluster } c \text { to its } i^{\text {th }} \text { user } \\
\text { on channel } l_{i}\end{array}$ \\
\hline$p_{i, l_{i}}^{c, j, b}$ & $\begin{array}{l}\text { Transmitted power to user } i \text { of cluster } c \text { on channel } I_{i} \\
\text { from } b^{\text {th }} \text { BS cluster } j\end{array}$ \\
\hline$\gamma_{i, l_{i}}^{c, b}$ & $\begin{array}{l}\text { SINR of user } i \text { in cluster } c \text { on channel } l_{i} \text { received from } \\
b^{\text {th }} \mathrm{BS} \text { of its cluster }\end{array}$ \\
\hline$\gamma_{i, l_{i}}^{c}$ & Total SINR of user $i$ in cluster $c$ on channel $l_{i}$ \\
\hline$\gamma_{\text {th }}$ & Minimum target SINR of users \\
\hline$p_{\max }$ & Maximum affordable transmitting power \\
\hline
\end{tabular}

BSs of a cluster, according to their location in the network. Namely, the nearer BS of the cluster to the cell-edge user is designed to provide larger part of target SINR of the user. So, we define a coefficient, $\beta_{i}^{c, b}$, associated to base station $b$ for user $i$ in cluster $c$ as:

$$
\beta_{i}^{c, b}=\frac{h_{i, l_{i}}^{c, c, b}}{\sum_{b^{\prime}=1}^{B} h_{i, l_{i}}^{c, c, b^{\prime}}} .
$$

Similar to central users, cell-edge ones are also allowed to request their own QoS level from the network. $\alpha_{i, c}$ is a factor assumed to be determined by cell-edge user $i$ belonging to cluster $c$. Indeed, $\beta_{i}^{c, b} \alpha_{i, c} \gamma_{t h}$ denotes the part of target SINR of user $i$ that should be provided by $b^{\text {th }}$ BS belonging to cluster $c$.

As all BSs belonging to the cluster transmit the needed information of one cell-edge user simultaneously on the same channel, the user receive total signal equal to:

$y_{i, c}=\frac{x_{i, c}}{\sqrt{\sigma_{x_{i, c}}^{2}}} \sum_{b=1}^{B} \sqrt{p_{i, l_{i}}^{c, c, b} h_{i, l_{i}}^{c, c, b}}+\sum_{k=1, k \neq c}^{C} \frac{x_{i, k}}{\sqrt{\sigma_{x_{i, k}}^{2}}} \sum_{b=1}^{B} \sqrt{p_{i, l_{i}}^{k, k, b} h_{i, l_{i}}^{k, k, b}}+n_{l_{i}}$

So the whole SINR of $i^{\text {th }}$ cell-edge user in cluster $c$ will be:

$$
\gamma_{i, l_{i}}^{c}=\sum_{b=1}^{B} \gamma_{i, l_{i}}^{c, b}=\sum_{b=1}^{B} \frac{p_{i, l_{i}}^{c, c, b} h_{i, l_{i}}^{c, c, b}}{I_{I . N .}^{i, c}}
$$

where $I_{I . N}^{i, c}$ is noise power density plus the received interference of user $i$ in cluster $c$ in channel $l_{i}$, caused by cell-edge users of other clusters using same channel as user $i$, and is defined as:

$$
I_{I . N}^{i, c}=I_{i, l_{i}}^{c}+\sigma_{0}^{2}=\sum_{j=1, j \neq c}^{C} \sum_{b=1}^{B} p_{i, l_{i}}^{c, j, b} h_{i, l_{i}}^{c, j, b}+\sigma_{0}^{2}
$$

\subsection{Stackelberg game model for cell-edge users}

As stated earlier, the objective of each BS is to provide only a fraction of cell-edge users' target SINR. On the other hand, since each user gets services from all BSs belonging to its cluster, each of them imposes different cost to the respected user proportional to the provided QoS. Therefore, by refining (3), the utility function of cell-edge user $i$ in cluster $c$ can be determined as:

$$
\begin{aligned}
U_{i}^{c}= & \alpha_{i, c}^{2} \operatorname{sgn}\left(\gamma_{i, l_{i}}^{c}-\alpha_{i, c} \gamma_{t h}\right) \log \left(1+\gamma_{i, l_{i}}^{c}\right) \\
& -\sum_{b=1}^{B}\left(\lambda_{i}^{c, b} p_{i, l_{i}}^{c, c b}+\varphi_{i}^{c, b}\right)+\frac{\gamma_{i, l_{i}}^{c}}{\alpha_{i, c} \gamma_{t h}} \mathrm{u}\left(\gamma_{i, l_{i}}^{c}-\alpha_{i, c} \gamma_{t h}\right),
\end{aligned}
$$

where $\lambda_{i}^{c, b}$ and $\varphi_{i}^{c, b}$ are the unit-price and the subscription imposed by BS $b$ of cluster $c$ to user $i$ in cell-edge. 
In turn, the utility function of each BS belonging to cluster $c$ is defined similar to (4) as follows:

$$
U_{B S}^{c, b}=\sum_{i=1}^{B \times N_{\text {out }}}\left(\lambda_{i}^{c, b} p_{i, l_{i}}^{c, c, b}+\varphi_{i}^{c, b}\right)-\sum_{i=1}^{B \times N_{\text {out }}}\left(\gamma_{i, l_{i}}^{c, b}-\beta_{i}^{c, b} \alpha_{i, c} \gamma_{\text {th }}\right)^{2} .
$$

Since each BS of one cluster serves all of the corresponding cell-edge users and the number of users in cell-edges of $B$ cells belonging to cluster $c$ is assumed to be $N_{\text {out }}$, the total number of users receiving services from each $B S$ in a cluster will be $B \times N_{\text {out }}$.

Similar to central region and with the same expectations, the imposed subscription from $b^{\text {th }}$ BS of cluster $c$ to cell-edge user $i$ is considered as:

$$
\varphi_{i}^{c, b}=\lambda_{i}^{c, b} \frac{I_{I, N .}^{i, c}}{h_{i, l_{i}}^{c, c, b}} .
$$

\subsection{Nash equilibrium of the extended game \\ 4.3.1 Existence of Nash equilibrium point in proposed Stackelberg game}

Obviously, the strategy space of cell-edge users is exactly the same as central ones. Therefore the concavity of utility functions with respect to their own strategies is the only necessary condition for existence of Nash equilibrium point for the extended game. by computing the secondorder partial derivative of (22) with respect to $p_{i, l_{i}}^{c, c, b}$, the following equation will be obtained:

$$
\frac{\partial^{2} U_{i}^{c}}{\partial\left(p_{i, l_{i}}^{c, c, b}\right)^{2}}=-\frac{\alpha_{i, c}^{2} h_{i, l_{i}}^{c, c, b}}{I_{I . N .}^{i, c}\left(1+\gamma_{i, l_{i}}^{c}\right)^{2}} .
$$

(25) is apparently always negative. Consequently, it is induced that the utility functions of each cell-edge user is strictly concave.

Eventually, Satisfying (26) leads to concavity of utility function of BSs determined in (23). So a pure Nash equilibrium point exists for the extended game too.

$$
\lambda_{i}^{c, b}<\frac{6 \alpha_{i, c}^{3} \beta_{i}^{c, b} \gamma_{t h} h_{i, l_{i}}^{c, c, b}}{I_{I . N .}^{i, c}\left(1+4 \alpha_{i, c} \beta_{i}^{c, b} \gamma_{t h}+4 \alpha_{i, c}^{2} \beta_{i}^{c, b} \gamma_{t h}^{2}\right)}+\frac{h_{i, l_{i}}^{c, c, b}}{I_{I . N .}^{i, c} \alpha_{i, c} \gamma_{t h}} .
$$

\subsubsection{Calculating available Nash equilibrium point}

Again for the same reason described in Section 3.2.1 the maximum utility of cell-edge user $i$ will be occurred when $\gamma_{i, l_{i}}^{c} \geq \alpha_{i, c} \gamma_{t h}$. Also as there would be no incentive for the service provider to deviate from the requested value of SINR, for cell-edge users we can approximately use $\gamma_{i, l_{i}}^{c, b}=\beta_{i}^{c, b} \gamma_{i, l_{i}}^{c}$ and modify utility function of cell-edge users. Then, by applying $\frac{\partial U_{i}^{c}}{\partial p_{i, l_{i}}^{c, c b}}=0$ in similar case, the allocated power to $i^{t h}$ user in cluster $c$ from BS $b$ of the cluster will be:

$p_{i, l_{i}}^{c, c, b}\left(\lambda_{i}^{c, b}\right)^{(k+1)}=\frac{\alpha_{i, c}^{2} \beta_{i}^{c, b}}{\lambda_{i}^{c, b(k+1)}-\frac{h_{i, l_{i}}^{c, c, b}}{I_{I, N .}^{i, c(k)} \alpha_{i, c} \gamma_{t h}}}-\left.\frac{\beta_{i}^{c, b} I_{I, N .}^{i, c(k)}}{h_{i, l_{i}}^{c, c, b}}\right|_{0} ^{P_{\max }}$

Substituting (28) in (23) and then optimizing the utility function of $b^{\text {th }}$ BS of cluster $c$ with respect to $\lambda_{i}^{c, b}$ will result in the following unit-price imposed to the $i^{t h}$ user:

$$
\lambda_{i}^{c, b(k+1)}=\frac{4 \alpha_{i, c}^{3} \beta_{i}^{c, b} \gamma_{t h} h_{i, l_{i}}^{c, c, b}}{I_{I . N .}^{i, c(k)}\left(1+4 \beta_{i}^{c, b} \alpha_{i, c} \gamma_{t h}+4 \beta_{i}^{c, b} \alpha_{i, c}^{2} \gamma_{t h}^{2}\right)}+\frac{h_{i, l_{i}}^{c, c, b}}{\alpha_{i, c} \gamma_{t h} I_{I . N .}^{i, c(k)}} .
$$

It is apparent that (28) satisfies the condition obtained in (26) and afterward the subscription $\varphi_{i}^{c, b}$ will be obtained as follow:

$$
\varphi_{i}^{c, b}=\frac{4 \alpha_{i, c}^{3}\left(\beta_{i}^{c, b}\right)^{2} \gamma_{t h} h_{i, l_{i}}^{c, c, b}}{1+4 \beta_{i}^{c, b} \alpha_{i, c} \gamma_{t h}+4 \beta_{i}^{c, b} \alpha_{i, c}^{2} \gamma_{t h}^{2}}+\frac{\beta_{i}^{c, b}}{\alpha_{i, c} \gamma_{t h}} .
$$

So total subscription imposed from service provider to cell-edge user is equal to:

$$
\varphi_{i}^{c}=\sum_{b=1}^{B} \varphi_{i}^{c, b}
$$

By substituting (29) in (30) and ignoring the negligible terms, the total subscription will be approximately:

$$
\varphi_{i}^{c}=\frac{4 \alpha_{i, c}^{3} \gamma_{t h}}{1+4 \alpha_{i, c} \gamma_{t h}+4 \alpha \gamma_{t h}^{2}}+\frac{1}{\alpha_{i, c} \gamma_{t h}} .
$$

which is exactly the same as imposed subscription to central users and obviously has all properties described for $\varphi_{i}^{m}$ in Section 3.2.1. Furthermore, it can be easily seen that the utility of the user is more than its target with a small deviation.

\subsubsection{Uniqueness of obtained Nash equilibrium point}

In order to evaluate the convergence of extended game to a unique Nash equilibrium point, three conditions mentioned in Section 3.2.1 should be satisfied. Considering our iterative power allocation expressed in (27), in order to satisfy the first condition (being positive), for the cell-edge user the following inequality should be satisfied:

$$
\lambda_{i}^{c, b} \leq\left(\alpha_{i, c}^{2}+\frac{1}{\alpha_{i, c} \gamma_{t h}}\right) \frac{h_{i, l_{i}}^{c, c, b}}{I_{I . N .}^{i, c} .} .
$$

According to (28), satisfying (32) results in $\alpha_{i, c}^{2}+$ $4 \beta_{i}^{c, b} \alpha_{i, c}^{4} \gamma_{t h}^{2} \geq 0$ which is always true. 
In order to satisfy the second condition, we show that updating functions are monotone, i.e., their first derivative with respect to imposed interference should become non-negative which conclude exactly the same as (32), and these are always satisfied.

Finally, considering $\boldsymbol{P}^{\prime}=\left\{p_{i, l_{i}}^{c, j, b} \mid 1 \leq i \leq N_{i n t}, 1 \leq c, j \leq C\right.$, $1 \leq b \leq B\}$, for $\forall \rho \geq 1$ we have $I_{I . N .}^{i, c(k)}\left(\rho \boldsymbol{P}^{\prime}\right) \leq$ $\rho I_{I . N .}^{i, c(k)}\left(\boldsymbol{P}^{\prime}\right)$ in cell-edge region. So with the same procedure described in Section 3.2.1 for central users, if we define:

$$
\mu_{i, c}=\frac{\alpha_{i, c}^{2}\left(1+4 \beta_{i}^{c, b} \alpha_{i, c} \gamma_{t h}+4 \beta_{i}^{c, b} \alpha_{i, c}^{2} \gamma_{t h}^{2}\right)}{4 \alpha_{i, c}^{3} \beta_{i}^{c, b} \gamma_{t h} h_{i, l_{i}}^{c, c, b}}-\frac{\beta_{i}^{c, b}}{h_{i, l_{i}}^{c, c, b}}
$$

which is non-negative due to positivity of allocated power level, we obviously have:

$$
\mu_{i, c} I_{I . N .}^{i, c}(\rho \boldsymbol{P}) \leq \mu_{i, c} I_{I . N .}^{i, c}(\boldsymbol{P}) \leq \rho \mu_{i, c} I_{I . N .}^{i, c}(\boldsymbol{P})
$$

Thus, the third condition is easily met. As a result, not only proposed algorithm has at least one pure Nash equilibrium point but it is also unique.

\section{Simulation results of proposed power allocation in static clusters}

In this section, we consider a cellular network consisting of 9 hexagonal cells, located in 3 static clusters each containing 3 cells with radius of $1 \mathrm{~km}$ shown in Fig. 2. Also, each BS serves 5 users in the central region whose radius is $2 / 3$ of cell radius and 3 users in cell-edges of its cluster.
Meanwhile, assuming path loss model, channel gains can be defined as [41]:

$$
h_{i, l_{i}}^{m, j}=\frac{A}{\left(d_{i, l_{i}}^{m, j}\right)^{n}}
$$

where $d_{i, l_{i}}^{m, j}$ denotes the distance of $i^{t h}$ user of cell $m$ to the BS of cell $j$. Path loss exponent $n$ is set to 3.6 and the value of constant $A$ is fixed to $7.75 \times 10^{-3}$. Furthermore, noise variance and $P_{\max }$ are considered to be $5 \times 10^{-15}$ and $1.5^{w}$ respectively for all users. Also $\gamma_{t h}$ is chosen to be 5 , while each user requests its target SINR by determining $\alpha$ from 1 to 2 which is set randomly in our simulations.

\subsection{Algorithm convergence and power consumption}

By simulating the power allocation algorithms for both central and cell-edge regions, the transmitted power to 5 central and 3 cell-edge users located in one cell are depicted in Fig. 3a and Fig. 3b respectively. The first thing can be deduced from them is the fast convergence of devised power allocation strategies in both regions. However, Fig. 3b shows that the proposed algorithm for cell-edge region is converged to its Nash equilibrium point relatively slower which is occurred after almost 15 iterations. Since both proposed games are finite, these convergences are regardless of the number of served users.

Meanwhile, low steady state level of power consumptions associated with users in the network is the second thing that can be educed from Fig. 3. As we observed after the algorithm converged, the level of powers allocated to users are in $\mathrm{mW}$ range.

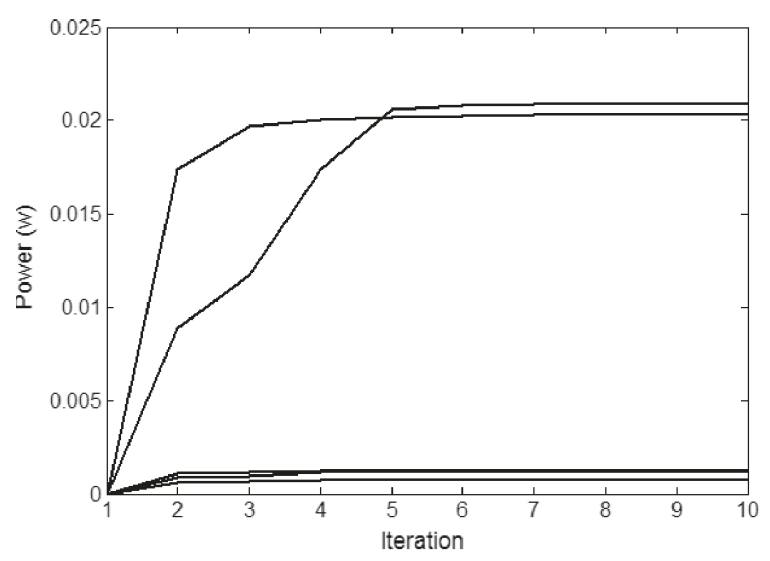

(a) Central region

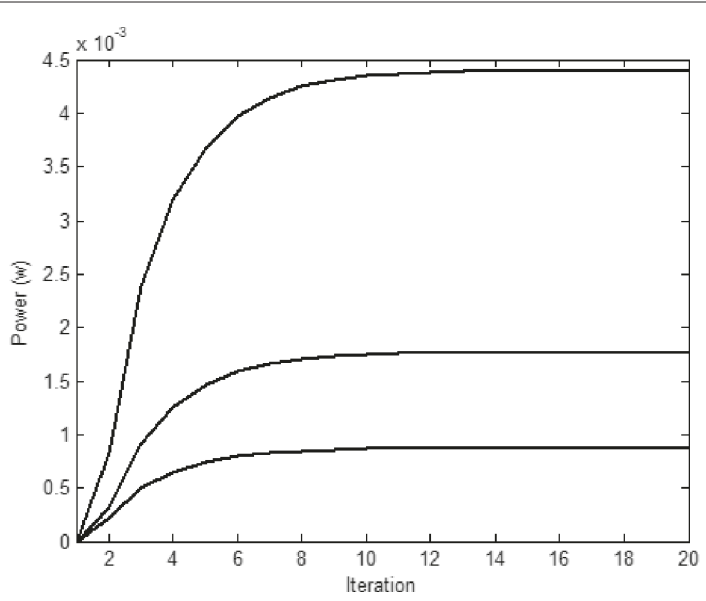

(b) Cell-edge region

Fig. 3 Convergence of power allocation algorithms. a Central region. b Cell-edge region 


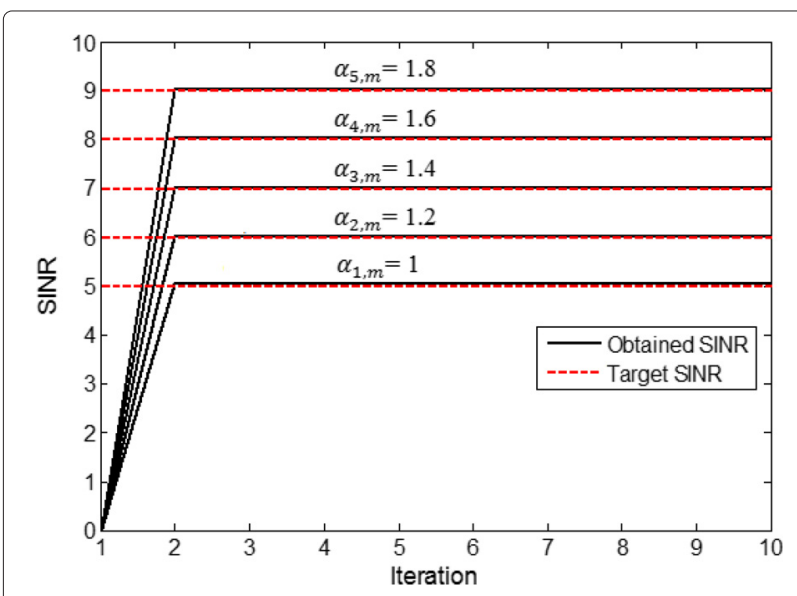

Fig. 4 Affording different requested SINRs for central users

\subsection{Affording the target QoS of users}

As discussed earlier, all users will request dynamic SINR threshold levels. In Fig. 4, it is apparent that the proposed power allocation method in central region is able to satisfy the requested demands of central users. Also, we consider the case that users request equal quality of services and the proposed algorithm for central users is now applied for the whole cell (both regions). As can be verified in Fig. 5a, for users close to the cell-edge, due to heavy ICI in addition to path loss effects, higher transmitting powers will be needed. It is shown in Fig. $5 \mathrm{~b}$ that if this kind of users request higher QoS, one BS may not be able to afford the required power by itself. Therefore, their requested SINR levels cannot be satisfied. Figure 6 demonstrates that the required transmitting power levels of cell-edge users can become affordable by coordinating three BSs of the cluster and applying CoMP-JT technique. So the network can provide even high QoS of users located in cell-edge region. These results clarify the necessity of clustering for cell-edge users.

Also, it can be elicited from Fig. 6a that the sum total level of power transmitted from 3 BSs to one cell-edge user is decreased in comparison with the needed transmitted power shown in Fig. 5a. The reason is that the signal sent from BSs of two closest neighbor cells are exchanged from interfering signal to useful ones. So the required power to provide the target SINR of the user is reduced.

\subsection{The effect of user's location in system performance}

In order to evaluate the amount improvement caused by making static clustering in use, we determined three users with different positions in our network, as illustrated in Fig. 7. User 1 is approximately located at the center of the cluster. User 3 that is far from other BSs of its cluster has the worst position in the network. The condition of user 2 is somehow between the conditions of two other users. Table 3 illustrates the power levels of three BSs in a cluster for each user. As expected, since neighboring cells are exchanged from interfere cells to useful ones, applied techniques decrease the level of needed power for all users. However, system performance is significantly improved for users in a position similar to user 1 . The coefficients $\beta$ for this user are $\left[\begin{array}{llll}0.2414 & 0.3187 & 0.4399\end{array}\right]$ which show that the target SINR is shared approximately equal between three BSs. In contrast, the system assigns [0.0138 0.01250 .9736$]$ as $\beta$ for user 3 which achieves close to requested SINR of BS of cell that contains this user. As a result, static clustering could not considerably decrease the required power for this kind of users in the network. For user 2, it is observed that the network performance becomes better than using no clustering but not as much as the performance improvement observed for user 1 .

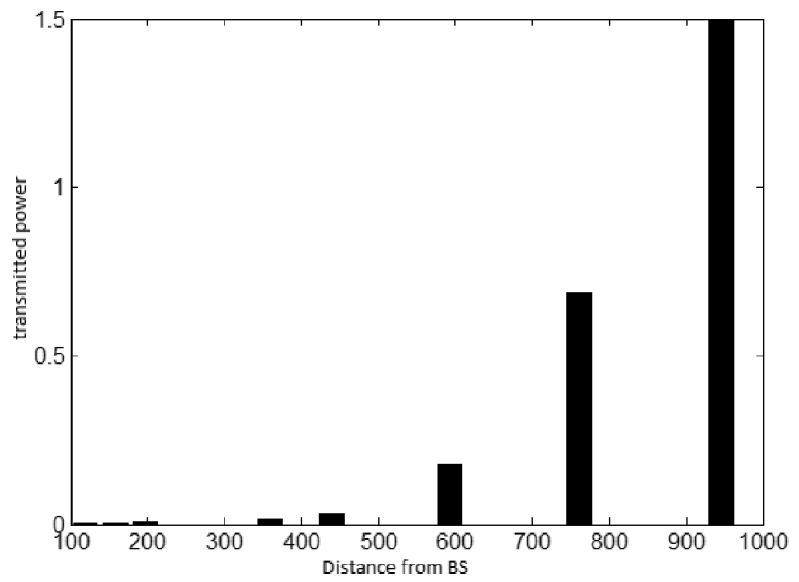

(a) Level of powers allocated to all users inside one cell.

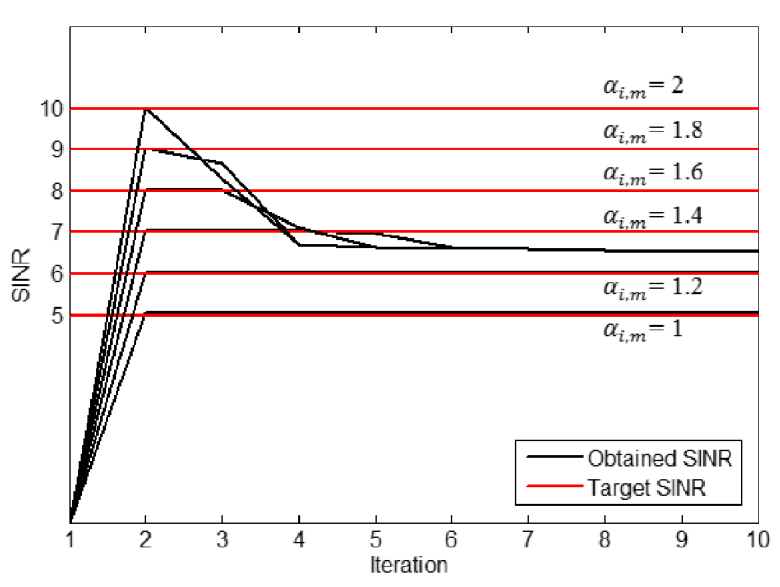

(b) Not affording high requested SINR anymore.

Fig. 5 Results without clustering. a Level of powers allocated to all users inside one cell. b Not affording high requested SINR anymore 


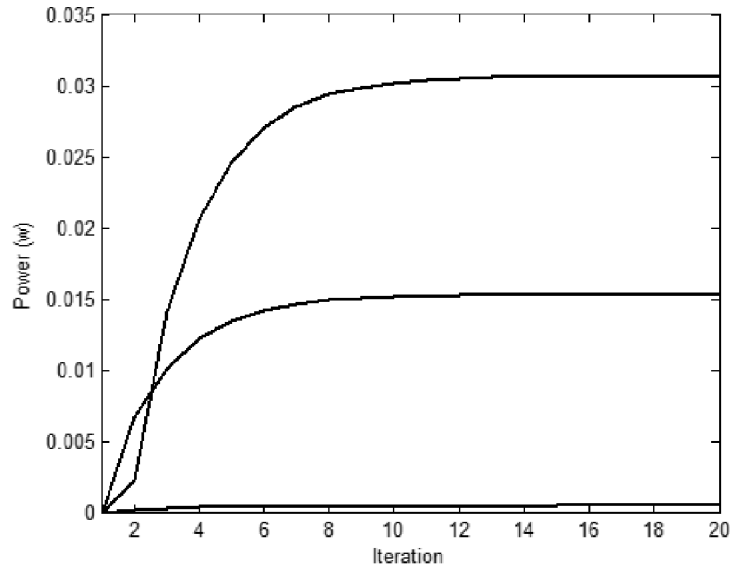

(a) Affording needed power level for cell-edge users.

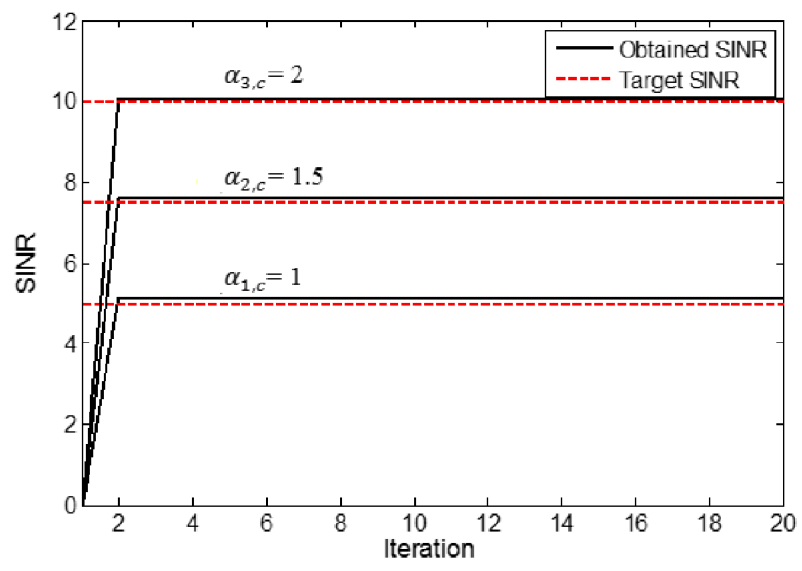

(b) Affording requested SINR for cell-edge users.

Fig. 6 Results with clustering. a Affording needed power level for cell-edge users. b Affording requested SINR for cell-edge users

\subsection{The effect of user's demand for QoS in imposed unit-price}

Figure 8a shows that the demand of one user to receive higher SINR (or equivalently higher QoS) will force the service provider to consider more unit-price for this user, as expected. This Figure is the same for both regions. Different unit-prices imposed to users based on their channel gains is shown in Fig. 8b, where it is observed that $\mathrm{BS} b$ of cluster $c$ imposes more unit-price for users with higher associated $\beta_{i}^{c, b}$. The reason of imposing unit-prices in such a way is that the revenue of the service provider with each established BS in the network is optimized.

\subsection{The effect of number of users in power consumption}

Finally it should be noted that since we consider an OFDMA cellular network where frequency bands are orthogonal and intra-call interference is ignored, increasing the number of users in central region or in cell-edge will not cause any changes on last results. In other words,

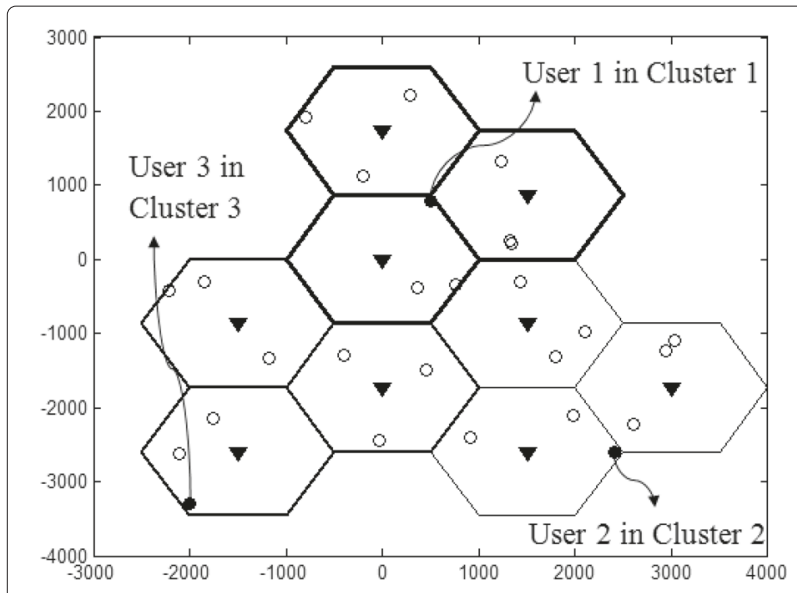

Fig. 7 Cell-edge users in the network with different positions in our architecture the number of users in each associated frequency band of central and cell-edge regions are equal to the number of cells and the number of clusters respectively, which are both constant. Consequently, the number of users in each cell will not have any effect on the number of users interfering with each other and in turn the level of needed powers will not change as well.

Figure 9 shows the effect of number of users in average power consumption. It is observed that although the network consumes higher level of power in average when it serves more users, but the effect of number of users in power consumption will become less as larger amount of users locate in network.

\subsection{Algorithm complexity}

In order to analyze the calculation complexity of developed scheme, the effect of number of cell-edge users in algorithm convergence is evaluated in Fig. 10. We observe that the number of iteration till convergence increases with the number of cell-edge user. This is indeed correlated to ICI levels experienced by each user. However, as the number of cell-edge users rises, the increasing rate of required iterations drops. In fact, according to Fig. 10 with 20 users in cell edges, the algorithm convergences after less than 80 iterations. Therefore, the proposed Stackelberg game reveals reasonable relationship between obtained advantages and the imposed complexity to the system.

Table 3 Power level consumption of three BSs in each cluster for all three users

\begin{tabular}{llll}
\hline & User 1 & User2 & User 3 \\
\hline BS 1 & 0.00876 & 0.0382 & $1.173 \times 10^{-6}$ \\
BS 2 & 0.00879 & $4.3499 \times 10^{-5}$ & $1.1858 \times 10^{-6}$ \\
BS 3 & 0.00884 & 0.0382 & 0.0138 \\
\hline
\end{tabular}




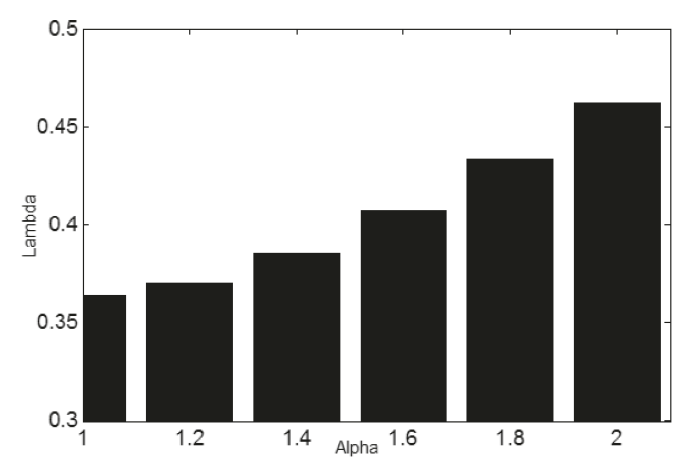

(a) User's demand for QoS in both regions.

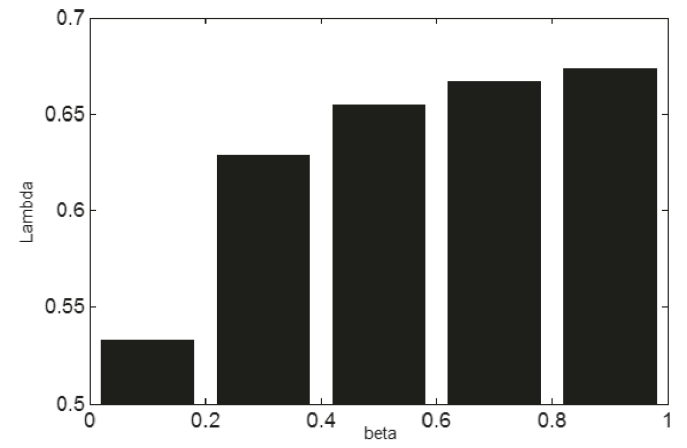

(b) $\beta$ that is determined by $\mathrm{BS}$ according to users' positions.

Fig. 8 Different unit-prices imposed to users corresponding to their demands for QoS and their locations. a User's demand for QoS in both regions. b $\beta$ that is determined by $\mathrm{BS}$ according to user's positions

\section{A dynamic clustering algorithm for inter-cell coordinated networks}

So far, we presented the resource allocation algorithm for a coordinated network which is statically clustered for cell-edge users. With respect to obtained results, despite being so helpful for users located near to center of predetermined clusters, static clustering cannot improve the system performance for all cell-edge users. In order to overcome this limitation, here we propose a dynamic clustering algorithm which enables the network to serve more cell-edge users with better available clusters. Indeed, the main goal in defining such dynamic algorithm is to show that conspicuous improvement in system performance can be achieved even by applying simple dynamic clusters.

In this section after introducing the considered system model, our proposed dynamic clustering algorithm is described. Finally simulation results for the proposed method of clustering are presented in which achieved improvement in system performance is evaluated.

\subsection{Proposed architecture for dynamic clustering model} We consider an OFDMA cellular network similar to what is shown in Fig. 11 which consists of $M$ cells. CoMP

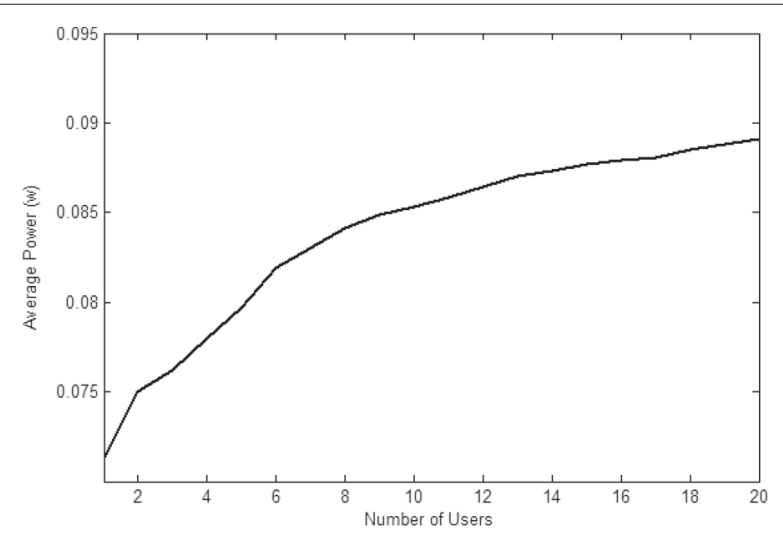

Fig. 9 The effect of number of users in power consumption functionalities are assumed to be available in the system, in a sense that central BSs of several cells could form the cluster and coordinate the transmission to serve users scattered randomly in edges of each cells. Clusters are considered to be disjointed in the network and each cell can belong to only one cluster. Also, in order to apply the power allocation algorithm for cell-edge users proposed in Section 4.2, the size of the clusters is supposed to be fixed to $B$. Also $D_{\max }$ denotes the maximum number of disjoint clusters containing $B$ cells that can be formed in the network.

\subsection{Proposed dynamic clustering algorithm}

In order to obtain the best possible clusters, we need to verify that the coordination of which BSs will yield the best performance for the network. As it is mentioned earlier, the base idea behind the proposed resource allocation algorithm presented in Section 4.2 is distributing the target SINR of the cell-edge user among coordinated BSs of the cluster according to their location in the network and applying CoMP-JT technique. According to the results obtained in Section 5, users who are nearer to the center of clusters are served in a better manner and the duty

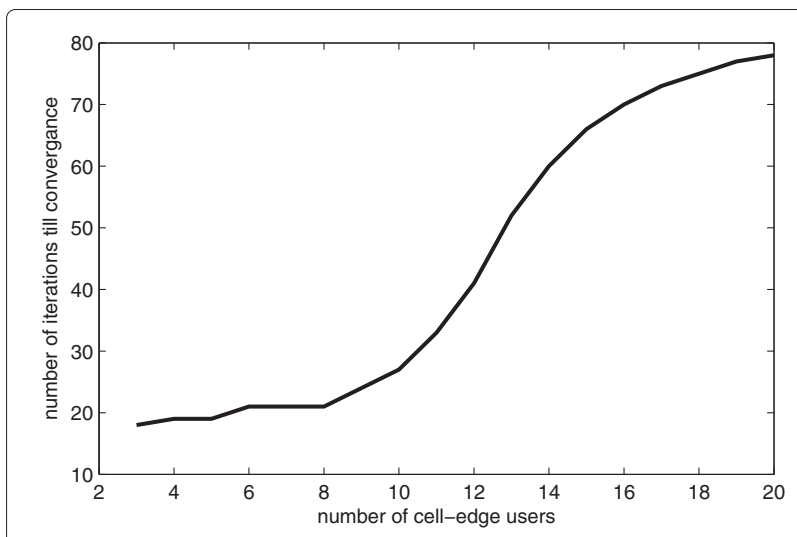

Fig. 10 Imposed calculation complexity to system 


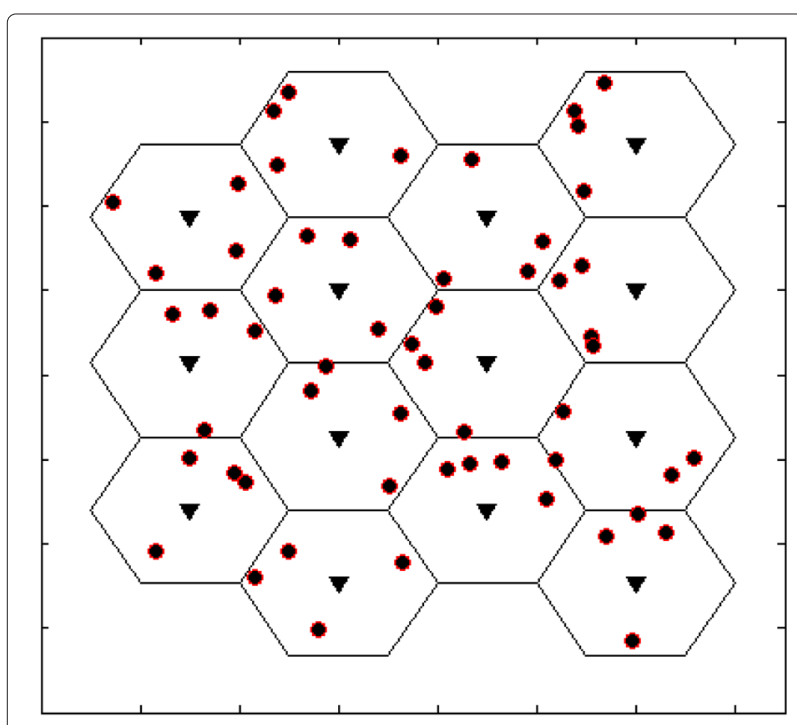

Fig. 11 System model for dynamic clustering: An OFDMA cellular network which consists of $\mathrm{M}$ cells

of affording their QoS is distributed better among coordinated BSs. Hence, users of this kind are located in a desirable situation in the network. One main result of Section 5 is that the BSs which have the strongest channel gains with the typical user are the best candidates to form its serving CoMP cluster. Furthermore, due to path loss effect, shorter distance between the user and the BS results in a better channel condition. By taking these facts into account, we ask each user to announce its requested cluster as a set of number of $B$ cells in which the distance criteria between cells' BSs and the user are least. Therefor, due to the report of a general user in the network, the $d^{\text {th }}$ cluster request is expressed as follow:

$$
C_{d}=\left\{\operatorname{Cell}_{d, 1}, \operatorname{Cell}_{d, 2}, \ldots, \operatorname{Cell}_{d, B}\right\} \quad d=1,2, \ldots, D_{\max }
$$

Hence, the collected information in the network will be presented as a list of pairs in the form of $\left.\left[C_{d}\right], N_{d}\right]$ in which $N_{d}$ is the number of events that $C_{d}$ has been requested to be formed by users. Undoubtedly, a cluster observed more in users' reports has got the higher potential to improve the system performance. But due to the presumption of having disjointed clusters, in some cases, simultaneous forming of clusters with the maximum number of requests might be impossible. So the decision must be taken in a way that the final clusters are not only the maximum requested ones, but there must be no common cells between them.

Let vector $\boldsymbol{N}=\left\{N_{1}, N_{2}, \ldots, N_{D_{\max }}\right\}$ denote the number of demands for making each possible cluster.
Also, the matrix $\boldsymbol{Y}$ with dimension $\mathrm{M} \times D_{\max }$ is defined as:

$$
y_{m, j}=\left\{\begin{array}{lll}
1 \operatorname{Cell}_{m} \in C_{j} & \\
& & \forall 1 \leq m \leq \mathrm{M} \\
0 & \text { o.w. } & \forall 1 \leq j \leq D_{\text {max }}
\end{array}\right.
$$

The proposed dynamic clustering method can be formulated in the form of an optimization problem as follows:

$$
\begin{array}{ll} 
& \max _{\boldsymbol{X}_{i}} \quad \boldsymbol{X}_{i} \times \boldsymbol{N} \\
\text { s.t. } & x_{i, j} \in\{0,1\} \quad \forall 1 \leq i, j \leq D_{\max } \\
& \sum_{j=1}^{D_{\max }} y_{m, j} x_{i, j} \leq 1,
\end{array}
$$

in which $\boldsymbol{X}_{i}=\left\{x_{i, 1}, x_{i, 2}, \ldots\right\}$ is the set of clusters which can be formed together. Indeed, the weights assigned to each available cluster in (38) equals to the corresponding element in vector $N$ which determines the number of requests by all users for each cluster. Under the second constraint in (38), the sets containing clusters with common cells are discarded. Obviously, the network will benefit more from forming the set of clusters with higher weights. So, among all possible sets, the one that maximizes the objective function of (38) under the mentioned constraints will be determined as the most requested disjoint clusters.

There are different kinds of techniques to solve the predetermined optimization problem. But in [32], a stepby-step algorithm is proposed to form the clusters with the aim of sum-capacity maximization. By considering our objectives and using similar approach, we propose a heuristic algorithm consisting of the following steps to find the most requested disjoint clusters instead of solving the optimization problem in (38):

1. Step 1: Specify the size of the cluster by setting the parameter $B$ and consider the null set of $C_{\text {opt }}$.

2. Step 2: Generate all available clusters with size $B$, or equivalently $C_{j} \quad \forall 1 \leq j \leq D_{\max }$, and the vector $\boldsymbol{N}$ containing the demands number associated to each cluster. Also, obtain the matrix $\boldsymbol{Y}$ according to (42).

3. Step 3: Define the square symmetric matrix $\boldsymbol{X}$ of size $D_{\max } \times D_{\max }$ as follows:

$$
x_{i, j}= \begin{cases}1 & i \neq j, C_{i} \cap C_{j}=\ddot{i}_{i} \frac{1}{2} \\ 1 & i=j \\ 0 & \text { o.w. }\end{cases}
$$

It means that if there exists a cell which belongs to both $C_{i}$ and $C_{j}$ clusters, the corresponding element 
of these clusters will be set to zero and it will be 1 if there is no common cell between them.

4. Step 4: Search for the maximum value in vector $\boldsymbol{N}$. Find the cluster for which this element is assigned to as the first CoMP cluster and add the index of cluster to $C_{\text {opt }}$. Then, set its corresponding element in vector $N$ to zero.

5. Step 5: Choose the $C_{d}$ cluster corresponding to $N_{d}$ which is the biggest element in modified vector $\boldsymbol{N}$ as the candidate for next CoMP cluster and set the element of $N_{d}$ in vector $\boldsymbol{N}$ to zero.

6. Step 6: Select the candidate cluster as the best one if (40) is satisfied and add its index to $C_{\mathrm{opt}}$. Otherwise, go back to step 5 .

$$
x_{d, j}=1 \quad \forall j \in C_{\mathrm{opt}}
$$

7. Step 7: Stop the algorithm if all elements in vector $\boldsymbol{N}$ are equal to zero. Otherwise, go back to step 5.

Finally, it should be noted that the best CoMP clusters can be obtained from the columns of $\boldsymbol{Y}$ matrix which their numbers exist in $C_{\text {opt }}$. Simulation results show that this proposed algorithm completely satisfies our purposes.

\subsection{Simulating the dynamic clustering algorithm}

In this section the effect of considering the position of users in opting the serving BSs is evaluated through clustering the cells by the proposed dynamic method. In other words, the resource allocation strategy presented in Section 4.2 is applied among users and the role of dynamic clustering in improving the system performance is clearly demonstrated through different simulations.

Since the goal of this section is comparing the performance of the network clustered by proposed dynamic method with the results of Section 5 which uses static clusters, the network architecture and system parameters are set with respect to Section 5. So we consider 3 cell-edge users located randomly in each cell of cellular network shown in Fig. 12. The size of each cluster is assumed to be 3. Namely, three coordinated BSs are clustered together and should provide the requested service of each user belonging to the cluster. Also in this paper according to the number of static clusters considered in Section 5 , the proposed power allocation algorithm is simulated in three disjoint dynamic clusters which are requested more than others. The channel model and the parameters of resource allocation algorithm are defined exactly the same as before.

By numbering the cells of network similar to Fig. 12, the $\boldsymbol{X}$ and $\boldsymbol{Y}$ matrices defined in (39) and (37) will be respectively obtained as (41) and (42). The first matrix shows all clusters consist of 3 cells which can be requested from users and the second one determines the possibility of

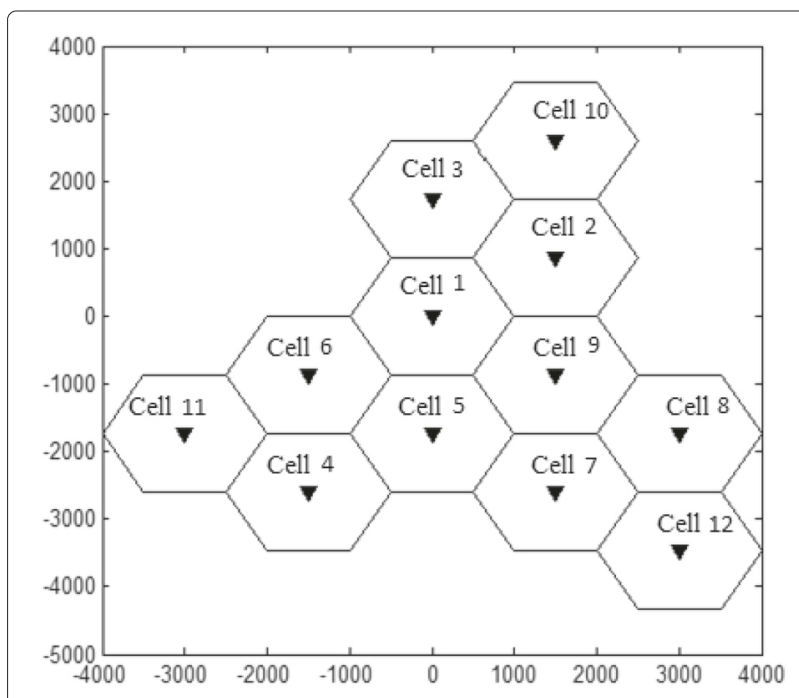

Fig. 12 Network model considered in simulations

forming them simultaneously according to disjoint clusters assumption.

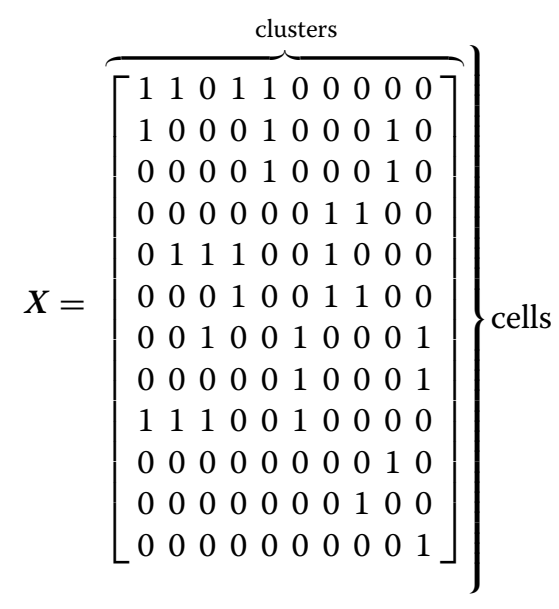

$$
Y=\overbrace{\left[\begin{array}{lllllllllll}
1 & 0 & 0 & 0 & 0 & 0 & 1 & 1 & 0 & 1 \\
0 & 1 & 0 & 0 & 0 & 0 & 0 & 1 & 1 & 1 \\
0 & 0 & 1 & 0 & 1 & 0 & 0 & 1 & 1 & 0 \\
0 & 0 & 0 & 1 & 0 & 1 & 0 & 0 & 1 & 1 \\
0 & 0 & 1 & 0 & 1 & 1 & 1 & 1 & 0 & 1 \\
0 & 0 & 0 & 1 & 1 & 1 & 1 & 1 & 1 & 0 \\
1 & 0 & 0 & 0 & 1 & 1 & 1 & 0 & 1 & 1 \\
0 & 1 & 1 & 1 & 0 & 1 & 1 & 1 & 1 & 1 \\
1 & 1 & 0 & 1 & 1 & 0 & 1 & 1 & 1 & 1
\end{array}\right]}^{\text {clusters }}\} \text { cells }
$$

\subsubsection{Cells clustering based on proposed dynamic algorithm}

The best disjoint clusters which are selected according to demands of users are depicted in Fig. 13b which is obtained By applying the proposed dynamic clustering algorithm to the predetermined network shown in Fig. 12. However Fig. 13a shows the static clusters considered 


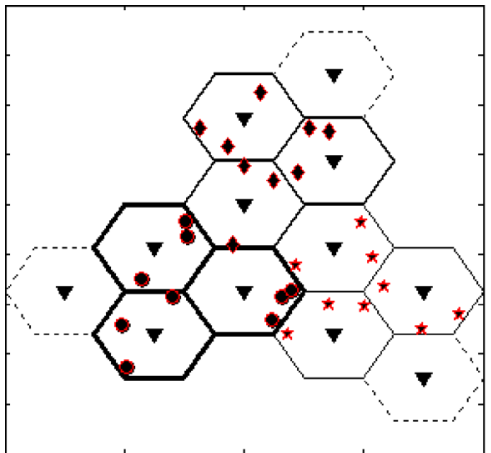

(a) Static clusters.

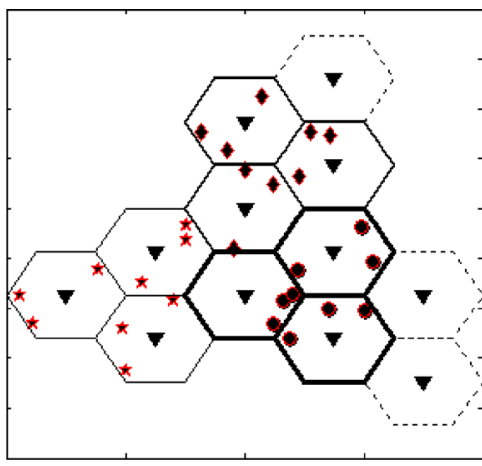

(b) Dynamic clusters

Fig. 13 Three clusters in which power allocation algorithm is applied. a Static clusters. b Dynamic clusters

in Section 5 for this configuration. In Fig. 13b most requested cluster are specified by darker lines. Indeed, by clustering the cells similar to Fig. 13b instead of applying static clusters, the number of users which are far away from two other coordinated BSs of the cluster will become minimum and the network can provide CoMP advantages for more users. As it is mentioned earlier despite static clustering, changing the number of each cell's users or their positions may lead the system to clusters reformation.

\subsubsection{The effect of clustering method in system performance} In order to evaluate the amount of helpful improvement caused by making dynamic clustering scheme in use, in this section we consider the typical user which is separated from others in Fig. 14. As it is shown in Fig. 14a, this user is far away from other BSs of its static cluster. The result of simulating the power allocation algorithm for cell edge users of static clusters is illustrated in Fig. 15a which shows that the needed power level of this user is approximately provided by the BS of its cell and two other coordinated BSs of the cluster have negligible role in affording the QoS of the user. In contrast, this user is approximately located at the center of the cluster formed in dynamic way as depicted in Fig. 14b. It is observed in Fig. 15b that the duty of providing target SINR of this user is shared nearly equal between three BSs belonging to the cluster and the needed transmitting power is distributed better between these coordinated BSs.

It should be noted again that although it is possible to find a user with better situation by making the static clusters in use, but number of this type of users will be less than the ones who benefit more from dynamic CoMP clustering. As the result, dynamic clustering method will be able to improve the performance of network and despite imposing higher level of complexity, is preferred to static clustering approach.

\subsubsection{The effect of clustering method in power consumption} By focusing on Fig. 15 it is understood that in the case of applying dynamic clustering, the total needed power transmitted from three coordinated BSs to the determined user is less than what is needed when clusters are static. Table 4 can explain the reason of such improvement

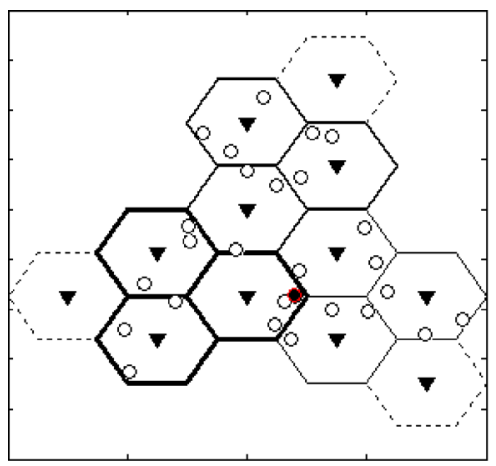

(a) Static clustering.

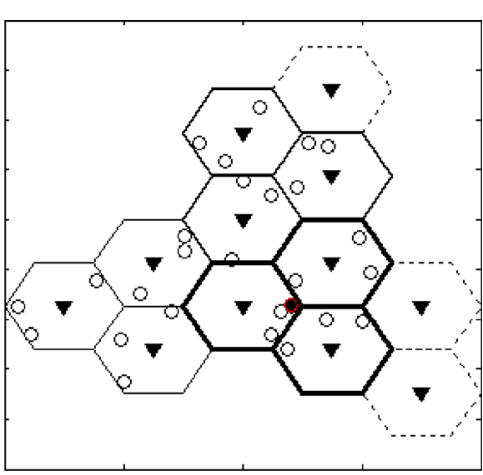

(b) Dynamic clustering

Fig. 14 The position of a typical user in its cluster. a Static clustering. b Dynamic clustering 


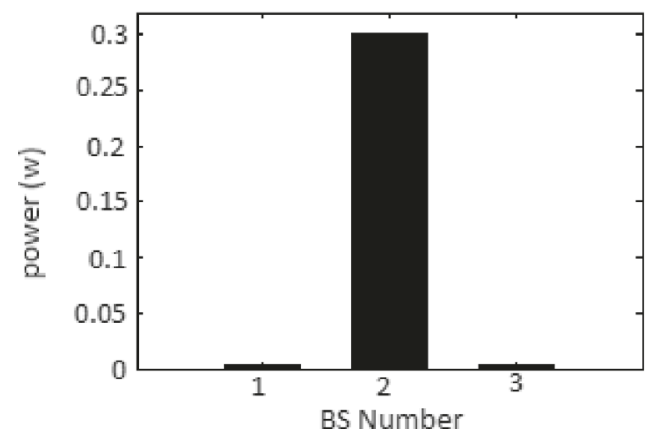

(a) Static clustering.

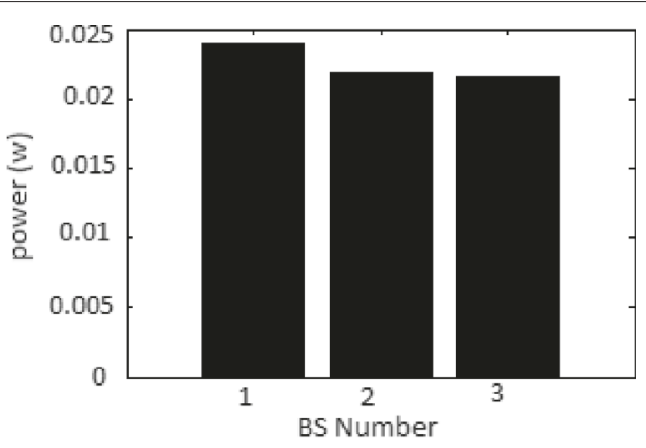

(b) Dynamic clustering.

Fig. 15 Power level consumption of three BSs of the cluster. a Static clustering. b Dynamic clustering

in power consumption. As it is observed if the clusters remain static this user is exposed to higher level of destructive interference than applying dynamic clusters and this is due to the fact that two neighbor cells of this user, that are interfering cells in case of using static clusters, are exchanged into useful ones when clusters are built dynamically.

The effect of clustering method in the level of power consumption of the system is evaluated in Fig. 16. As it is depicted in Fig. 16a when clusters are formed dynamically most of the BSs need to transmit lower level of average power to provide the requested services of their users in comparison with static clustering. In this Fig. three first cells belong to the most requested cluster. Also according to Fig. 16b the average needed power of the network to afford the demand of all users will be decreased by making the proposed dynamic clustering method in use.

\subsubsection{The effect of increasing number of users in power consumption}

The last issue to be considered in our assessments is the effect of variation in the number of users located in edges of each cell. Figure 17 shows that regardless of clustering method, the network needs to consume higher level of power in average by increasing the number of each cell's users, as it is expected. Furthermore, for equal number of users, the average needed power of the network clustered dynamically will never be more than the network containing static clusters and the equal state is occurred when the best possible clusters are exactly the same as static ones applied in Section 5.

Table 4 The interference imposed to the specified user

\begin{tabular}{ll}
\hline Clustering method & Interference level \\
\hline Statically & $19.3095 \times 10^{-14}$ \\
Dynamically & $4.291 \times 10^{-14}$ \\
\hline
\end{tabular}

\section{Conclusions}

Since in conventional cellular networks, performance of near-edge users may be degraded due to strong interference, CoMP-JT is recently introduced as a promising technology to address such issues. In this paper, we proposed a new power allocation algorithm based on game theory for multi-cell networks. In our model, users are separated according to their distances from BSs of cells they are located in. In order to improve the condition of users in cell-edges, lower channel reuse factors are assigned to them through clustering and applying CoMPJT technology. As a result, dynamic target SINR levels are adopted for different users and by applying a Stackelberg game model, utility of users and revenue of the service providers are concurrently optimized.

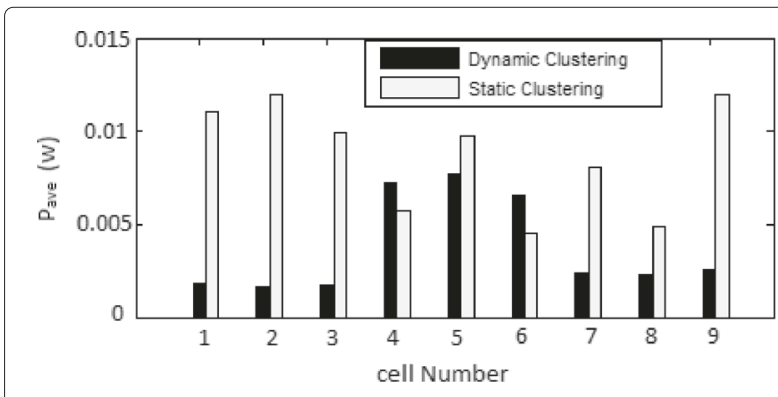

(a) Consumed in each cell

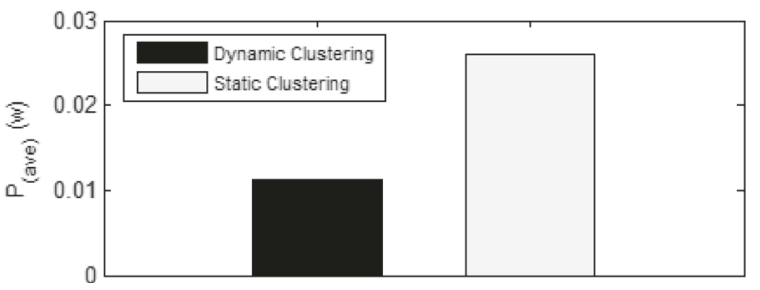

(b) Consumed in network.

Fig. 16 The effect of clustering method in level of average transmitting power. a Consumed in each cell. b Consumed in network 


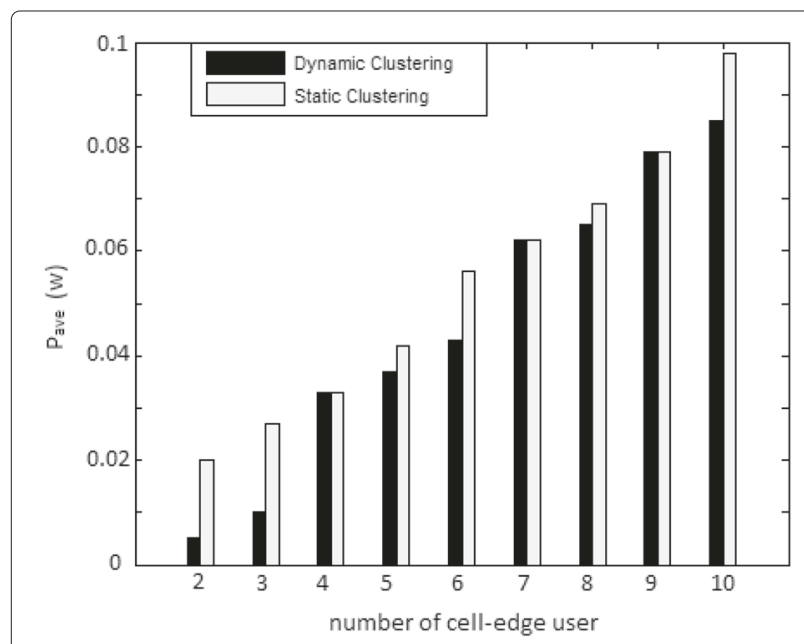

Fig. 17 The effect of increasing the number of users on the level of average transmitting power

Due to random channel association model used in this paper, the utility of users does not directly depend on allocated frequency channels. Therefore, one direction for future work is to provide a game theory based approach for joint channel assignment and power allocation in coordinated multi-cell networks. User mobility is also another issue that can be addressed in future extensions of this work.

\section{Competing interests}

The authors declare that they have no competing interests.

\section{Acknowledgment}

The authors would like to thank the anonymous reviewers for their helpful comments. This research was in part supported by a grant from IPM and also partially supported by Iran National Science Foundation (INSF) under contract No. 92/32575.

\section{Author details}

${ }^{1}$ Department of Electrical Engineering, Sharif University of Technology, Tehran, Iran. ${ }^{2}$ School of Computer Science, Institute for Research in Fundamental Sciences (IPM), Tehran, Iran.

Received: 11 May 2015 Accepted: 2 March 2016

Published online: 01 April 2016

\section{References}

1. G Boudreau, J Panicker, N Guo, R Chang, N Wang, S Vrzic, Interference coordination and cancellation for $4 \mathrm{G}$ networks [LTE part II: 3GPP release 8]. IEEE Commun. Mag. 47(4), 74-81 (2009)

2. G Fodor, C Koutsimanis, A Racz, N Reider, Intercell interference coordination in OFDMA networks and in the 3GPP long term evolution system. J. Commun. 4(7), 74-81 (2009)

3. 3GPP, Physical layer aspect for evolved Universal Terrestrial Radio Access (UTRA). TR 25.814, 3rd Generation Partnership Project (3GPP) (October 2006)

4. H Lei, L Zhang, D Yang, in Proc. 18th International Symposium on Personal, Indoor and Mobile Radio Communications (PIMRC). A novel multi-cell OFDMA systems structure using fractional frequency reuse (IEEE, Athens, Greece, 2007), pp. 1-5

5. R Irmer, H Droste, P Marsch, M Grieger, H Fettweis, S Brueck, H Mayer, L Thiele, V Jungnickel, Coordinated multipoint: Concepts, performance, and field trial results [IMT-Advanced and next-generation mobile networks]. IEEE Commun. Mag. 46(2), 102-111 (2011)

6. M Karakayali, G Foschini, R Valenzuela, Network coordination for spectrally efficient communications in cellular systems. IEEE Wireless Commun. Mag. 13(4), 56-61 (2006)

7. F Boccardi, H Huang, in Proc. 18th IEEE International Symposium on Personal, Indoor and Mobile Radio Communications (PIMRC). Limited downlink network coordination in cellular networks (IEEE, Athens, Greece, 2007)

8. S Brueck, L Zhao, J Giese, MA Amin, in Proc. 2010 International ITG Workshop on Smart Antennas (WSA). Centralized scheduling for joint transmission coordinated multi-point in LTE-Advanced (IEEE, Bremen, Germany, 2010), pp. 177-184

9. P Marsch, G Fettweis, Uplink comp under a constrained backhaul and imperfect channel knowledge. IEEE Trans. Wireless Commun. 10(6), 1730-1742 (2011)

10. P Marsch, G Fettweis, in Proc. IEEE International Conference on Communications (ICC). Static clustering for cooperative multi-point (comp) in mobile communications (IEEE, Kyoto, Japan, 2011), pp. 1-6

11. U Jang, H Son, J Park, S Lee, Comp-csb for ici nulling with user selection. IEEE Trans. Wireless Commun. 10(9), 2982-2993 (2011)

12. GY Li, J Niu, D Lee, J Fan, Y Fu, Multi-cell coordinated scheduling and MIMO in LTE. IEEE Commun. Surv. Tutor. 16(2), 761-775 (2014)

13. DWK Ng, ES Lo, R Schober, Energy-efficient resource allocation in multi-cell OFDMA systems with limited backhaul capacity. IEEE Trans. Wireless Commun. 11(10), 3618-3631 (2012)

14. AH Sakr, H ElSawy, E Hossain, in Proc. IEEE International Conference on Communications (ICC). Location-aware coordinated multipoint transmission in OFDMA networks (IEEE, Sydney, Australia, 2014), pp. 5166-5171

15. E Yaacoub, Z Dawy, A survey on uplink resource allocation in OFDMA wireless networks. IEEE Commun. Surv. Tutor. 14(2), 322-337 (2011)

16. G Hosseinabadi, Downlink channel assignment and power control for cognitive radio networks using game theory. Technical Report (2007)

17. L Zhou, RQ Hu, Y Qian, HH Chen, Energy-spectrum efficiency tradeoff for video streaming over mobile ad hoc networks. IEEE J. Selected Areas Commun. 31(5), 981-991 (2013)

18. XXu, T Qiu, W Xu, Z He, K Niu, in Proc. IEEE International Conference on Network Infrastructure and Digital Content (IC-NIDC). Sub-carrier allocation combined with coordinated multi-point transmission in multi-cell ofdma system (IEEE, Beijing, China, 2009), pp. 842-846

19. T Zhang, C Feng, G Su, in Proc. 5th International Conference on Wireless Communications, Networking and Mobile Computing, WiCom. Uplink multi-cell non-cooperative power allocation game algorithm for OFDMA cellular networks (IEEE, Beijing, China, 2009), pp. 1-4

20. UO Candogan, I Menache, A Ozdaglar, PA Parrilo, in Proc. IEEE INFOCOM. Near-optimal power control in wireless networks: A potential game approach (IEEE, San Diego, CA, 2010), pp. 1954-1962

21. Z Liang, YH Chew, CC Ko, in Proc. IEEE Global Telecommunications Conference, GLOBECOM. On the modeling of a non-cooperative multi-cell OFDMA resource allocation game with integer bit-loading (IEEE, Hawaii, USA, 2009), pp. 1-6

22. F Huang, S Wang, S Du, in Proc. 20th Annual Wireless and Optical Communications Conference (WOCC). Resource allocation in OFDM-based multi-cell cognitive radio systems (IEEE, San Diego, USA, 2011), pp. 1-5

23. L Zhou, M Chen, Y Qian, HH Chen, Fairness resource allocation in blind wireless multimedia communications. IEEE Transactions on Multimedia. 15(6), 946-956 (2013)

24. G Zhang, C Li, E Ding, K Yang, X Yang, in Processing of IEEE International Conference on Communications (ICC). Fair and efficient resource sharing for selfish cooperative communication networks using cooperative game theory (IEEE, Kyoto,Japan, 2011), pp. 1-5

25. H von Srackelberg, Market Structure and Equilibrium. (Springer, 1934)

26. J Zhang, Q Zhang, in Proc. of the 10th ACM International Symposium on Mobile Ad Hoc Networking and Computing. Stackelberg game for utility-based cooperative cognitive radio networks (ACM, New York, USA, 2009), pp. 23-32

27. A Daoud, T Alpcan, S Agarwal, M Alanyali, in Proc. 47th IEEE Conference on Decision and Dontrol, CDC. A stackelberg game for pricing uplink power in wide-band cognitive radio networks, (Cancun, Mexico, 2008), pp. 1422-1427 
28. N Omidvar, BH Khalaj, in Proc. IEEE 16th International Workshop on Computer Aided Modeling and Design of Communication Links and Networks (CAMAD). A game theoretic approach for power allocation in the downlink of cognitive radio networks (IEEE, Kyoto, Japan, 2011), pp. 158-162

29. S Venkatesan, in Processing of 18th IEEE International Symposium on Personal, Indoor and Mobile Radio Communications (PIMRC). Coordinating base stations for greater uplink spectral efficiency in a cellular network (IEEE, Athens, Greece, 2007), pp. 1-5

30. M Kamoun, L Mazet, in Processing of 8th IEEE Workshop on Signal Processing Advances in Wireless Communications (SPAWC). Base-station selection in cooperative single frequency cellular network (IEEE, Helsinki, Finland, 2007), pp. 1-5

31. J Li, C Botella, T Svensson, Resource allocation for clustered network MIMO OFDMA systems. EURASIP J. Wireless Commun Networking (2012)

32. A Papadogiannis, D Gesbert, E Hardouin, in Processing of IEEE International Conference on Communications (ICC). A dynamic clustering approach in wireless networks with multi-cell cooperative processing (IEEE, Beijing, China, 2008), pp. 4033-4037

33. A Papadogiannis, GC Alexandropoulos, in Processing of IEEE International Conference on Fuzzy Systems (FUZZ). The value of dynamic clustering of base stations for future wireless networks (IEEE, Barcelona, Spain, 2010), pp. 1-6

34. K Maruta, T Maruyama, A Ohta, M Nakatsugawa, in Processing of 20th IEEE Personal, Indoor and Mobile Radio Communications. Inter-cluster interference canceller for multiuser mimo distributed antenna systems (IEEE, Tokyo, Japan, 2009), pp. 3079-3083

35. S Kaviani, W Krzymien, in Processing of the IEEE Global Telecommunications Conference (GLOBECOM). Multicell scheduling in network mimo (IEEE, Miami, Florida, USA, 2010), pp. 1-5

36. A Papadogiannis, HJ Bang, D Gesbert, E Hardouin, Efficient selective feedback design for multicell cooperative networks. 60(1), 196-205 (2011)

37. S Fu, B Wu, PH Ho, X Ling, in Processing of IEEE International Conference on Communications (ICC). Interference coordination in comp with transmission scheduling and game theoretical power reallocation (IEEE, Ottawa, Canada, 2012), pp. 4212-4217

38. LA Petrosjan, NA Zenkevich, Game Theory. (Singapore; River Edge, N. J.; World Scientific, Series on Optimization 3., 1996)

39. S Boyd, L Vandenberghe, Convex Optimization. (Cambridge University Press, New York, NY, USA, 2004)

40. RD Yates, A framework for uplink power control in cellular radio systems. IEEE J. Selected Areas Commun. 13(7), 1341-1347 (1995)

41. V Shah, NB Mandayam, DJ Goodman, in Proc. IEEE Int. Symp. on Personal, Indoor and Mobile Radio Communications (PIMRC). Power control for wireless data based on utility and pricing (IEEE, Osaka, Japan, 1998), pp. 1427-1432

\section{Submit your manuscript to a SpringerOpen ${ }^{\circ}$ journal and benefit from:}

- Convenient online submission

- Rigorous peer review

- Immediate publication on acceptance

- Open access: articles freely available online

- High visibility within the field

- Retaining the copyright to your article

Submit your next manuscript at springeropen.com 